\title{
VULNERABILIDAD DE ESTRUCTURAS CON BASE EN MARCOS DE CONCRETO REFORZADO EN EL VALLE DE MÉXICO ANTE UN ESCENARIO SIMILAR AL SISMO DE SEPTIEMBRE DE 1985*
}

\author{
Eber Alberto Godínez Domínguez ${ }^{(1)}$, Arturo Tena Colunga ${ }^{(1)}$ y Luis Eduardo Pérez Rocha ${ }^{(2)}$
}

\begin{abstract}
RESUMEN
Se presenta una estimación de la respuesta no lineal de estructuras con base en marcos de concreto reforzado en el Distrito Federal, con la finalidad de realizar un estudio de su vulnerabilidad sísmica mediante la construcción de mapas de isodemandas (ductilidad y desplazamiento), considerando diferentes periodos estructurales y recreando, en lo posible, el escenario sísmico correspondiente al sismo del 19 de septiembre de 1985. El comportamiento no lineal de las estructuras de concreto se representó utilizando el modelo de histéresis propuesto por Takeda. Las resistencias nominales ante carga lateral de las estructuras de concreto existentes en el valle de México se estimaron conforme a las mínimas asociadas a un diseño conforme a los reglamentos de construcción que estuvieron vigentes antes del sismo de 1985: RCDF-42, RCDF-57, RCDF-66 y RCDF-76. Se estimó también la sobrerresistencia probable asociada a estos sistemas estructurales. Posteriormente, los contornos de isodemandas obtenidos se comparan con la ubicación geográfica de los daños observados durante los sismos de septiembre de 1985.
\end{abstract}

\begin{abstract}
This paper presents vulnerability maps for reinforced concrete moment frames (RCMFs) in Mexico City's metropolitan area for $\mathrm{a}_{\mathrm{s}}=8.1$ earthquake scenario assessing peak nonlinear dynamic responses using the concept of displacement ductility demand spectra (DDDS). The considered earthquake scenario is an equivalent scenario to the $\mathrm{M}_{\mathrm{s}}=8.1$ September 19, 1985 Michoacán Earthquake that severely affected Mexico City. The Takeda model was used to simulate the nonlinear seismic behavior of RCMFs. Nominal strength under lateral loading for the existing RCMFs structures in Mexico's City during the 1985 earthquake was estimated according to the nominal strength required by previous version of Mexico's Federal District Code that were effective before the 1985 earthquake. Overstrength sources associated to RCMFs were also evaluated. These vulnerability maps were compared with the recognized geographic location of heavy structural damage and collapses observed during the 1985.
\end{abstract}

* Artículo derivado de la tesis ganadora del Cuarto Concurso Nacional de Tesis de Maestría organizado por la Sociedad Mexicana de Ingeniería Sísmica. Recibido el 29 de septiembre de 2005 y aprobado para su publicación el 25 de octubre de 2005. Se aceptarán comentarios y/o discusiones hasta cinco meses después de su publicación.

(1) Universidad Autónoma Metropolitana Azcapotzalco, Departamento de Materiales, Av. San Pablo 180, Col. Reynosa Tamaulipas, 02200, México, D.F.eagd@correo.azc.uam.mx, atc@correo.azc.uam.mx

(2) Instituto de Investigaciones Eléctricas, Calle Reforma 113, Col. Palmira, 62490, Cuernavaca, Morelos. lepr@iie.org.mx 


\section{INTRODUCCIÓN}

Una de las principales preocupaciones en la ingeniería sísmica ha sido el estimar de una manera sencilla la respuesta no lineal de las estructuras ante sismos intensos. Lo anterior debido a que la mayoría de las estructuras se comportan inelásticamente durante eventos sísmicos de estas características, principalmente en aquéllos de larga duración como los que se presentaron en la Ciudad de México en septiembre de 1985.

Se presenta una estimación de la respuesta no lineal de estructuras de concreto reforzado en el Distrito Federal, con la finalidad de realizar un estudio de su vulnerabilidad sísmica mediante la construcción de mapas de isodemandas (ductilidad y desplazamiento), considerando diferentes periodos estructurales y recreando, en lo posible, el escenario sísmico correspondiente al sismo del 19 de septiembre de 1985 a partir de los registros instrumentales y las funciones de transferencia promedio obtenidas a partir de los registros de la red acelerográfica del Valle de México. El comportamiento no lineal de las estructuras de concreto se representó utilizando el modelo de histéresis propuesto por Takeda. Las resistencias nominales ante carga lateral de las estructuras de concreto existentes en el Valle de México se estimaron conforme a las mínimas asociadas a un diseño conforme a los reglamentos de construcción que estuvieron vigentes antes del sismo de 1985: RCDF-42, RCDF-57, RCDF-66 y RCDF-76. Se valoró también la sobrerresistencia esperada en estos sistemas estructurales. Posteriormente, los contornos de isodemandas obtenidos se compararon con la ubicación geográfica de los daños observados durante los sismos de septiembre de 1985. Los parámetros de estudio, principalmente las demandas de ductilidad, derivadas del análisis dinámico no-lineal de sistemas de un grado de libertad, introducen conceptos que permiten describir la capacidad destructiva de un evento sísmico.

La metodología propuesta, mediante los mapas de isodemandas, permite identificar las zonas con niveles de demandas elevados, es decir, aquéllas que pueden ser vulnerables ante un evento sísmico intenso semejante al del 19 de septiembre de 1985. La correlación obtenida entre las zonas de demandas elevadas y la de mayores daños observados en 1985 es razonable. Además, este procedimiento permite ubicar zonas de demandas altas para distintos sistemas estructurales, por lo que puede ser muy útil para guiar estudios de planeación urbana y de regulación del uso del suelo en la zona metropolitana de la Ciudad de México.

\section{ESTUDIOS PREVIOS}

El sismo del 19 de septiembre de 1985 ha sido uno de los más destructivos en la historia de la Ciudad de México debido a su gran magnitud y duración. Otra causa importante que contribuyó a su gran destructividad fueron los efectos de sitio debidos a las condiciones del subsuelo en la Ciudad de México, dado que en algunas zonas de terreno blando las ondas presentaron niveles de amplificación importantes, lo que ocasionó daño severo en numerosas estructuras. En la Ciudad de México el sismo de 1985 se registró únicamente en 11 estaciones acelerográficas (figura 1): los sitios Tacubaya (TACY) y Ciudad Universitaria (CU01, CUIP y CUMV) están localizados sobre terreno firme, Viveros de Coyoacán (SXVI) sobre suelos de transición, y el resto en cuatro 
puntos diferentes de la zona de lago: la Secretaría de Comunicaciones y Transportes (SCT1), Tláhuac (TLHB y TLHD), Central de Abastos (CDAO y CDAF) y Texcoco Sosa (TXSO). La escasa instrumentación permitió únicamente un ligero entendimiento de cómo los efectos de sitio se relacionaron con los daños observados en las estructuras existentes. Asimismo, fue difícil correlacionar el daño observado con los registros acelerométricos observados.

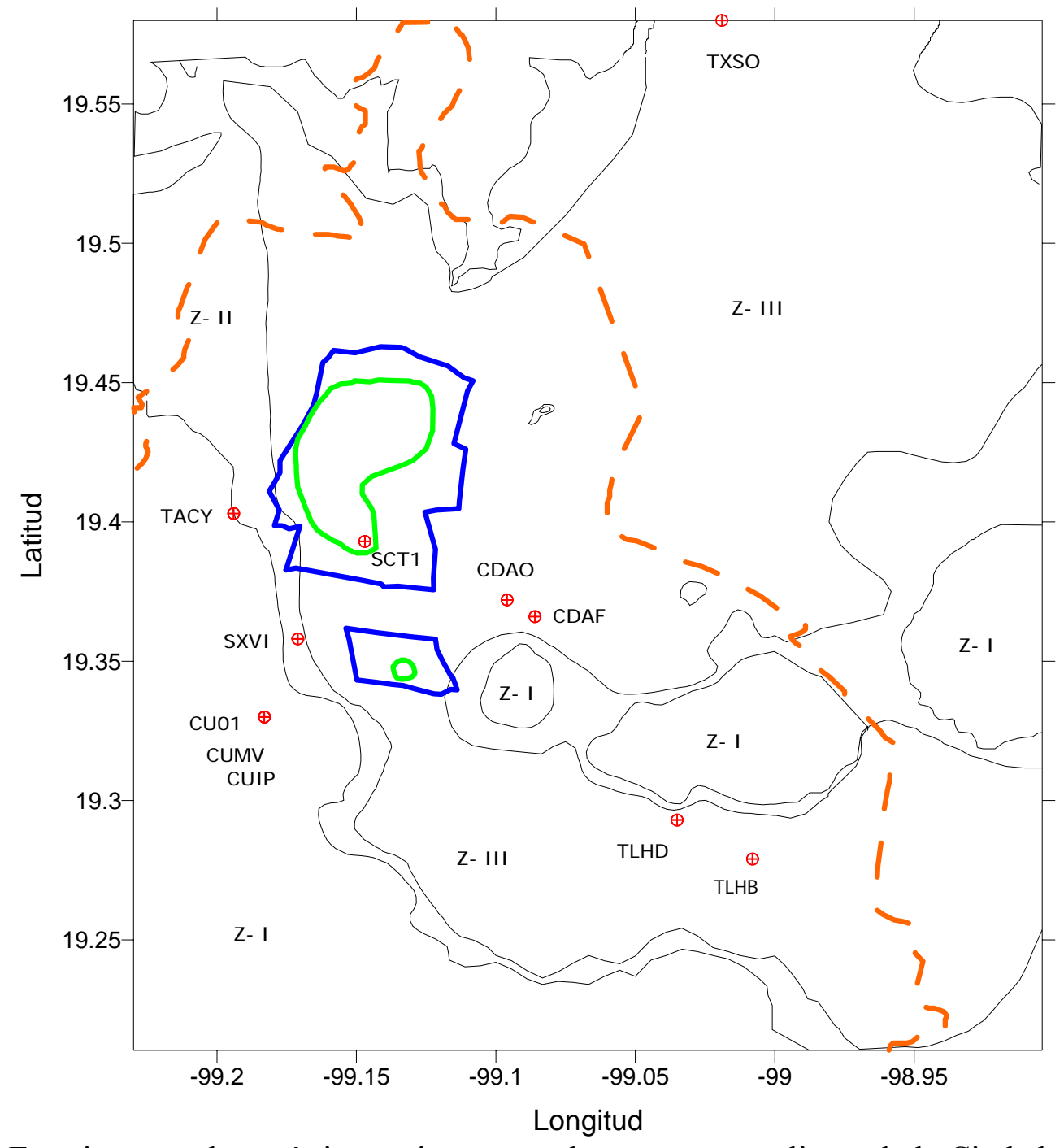

Figura 1. Estaciones acelerométricas existentes en la zona metropolitana de la Ciudad de México durante el sismo del 19 de septiembre de 1985. Las zonas con mayores daños se muestran en líneas azul y verde. El contorno del Distrito Federal se ilustra en línea discontinua de color naranja.

De las estaciones ubicadas en la zona de lago, sólo SCT1 se localizaba en la zona de grandes daños. Las cinco estaciones restantes estaban localizadas en zonas donde no se observaron daños severos en edificios (en Tláhuac se reportaron daños en las tuberías de abastecimiento de agua potable). De hecho, no existían suficientes estaciones acelerométricas en la zona que presentó mayor densidad de colapsos, dichas zonas contemplan las zonas marcadas 
con línea verde en la figura 1, donde se ubican las colonias Roma, Condesa y el centro de la Ciudad de México. Dado que, conforme al estado del conocimiento de 1985, las características de las estructuras y del suelo en las zonas cercanas a SCT eran similares a aquéllas de las colonias Roma y Condesa, fue difícil explicar por qué la densidad de colapsos observados en estas últimas dos colonias fue mayor que cerca de SCT. Por lo tanto, resultó claro que existía la imperiosa necesidad de realizar un programa de densificación de la instrumentación sísmica de la ciudad de México, entre otras razones: (a) para entender mejor lo ocurrido durante los sismos de septiembre de 1985 y, (b) depurar la zonificación sísmica y los espectros de diseño para las distintas zonas geotécnicas de la ciudad.

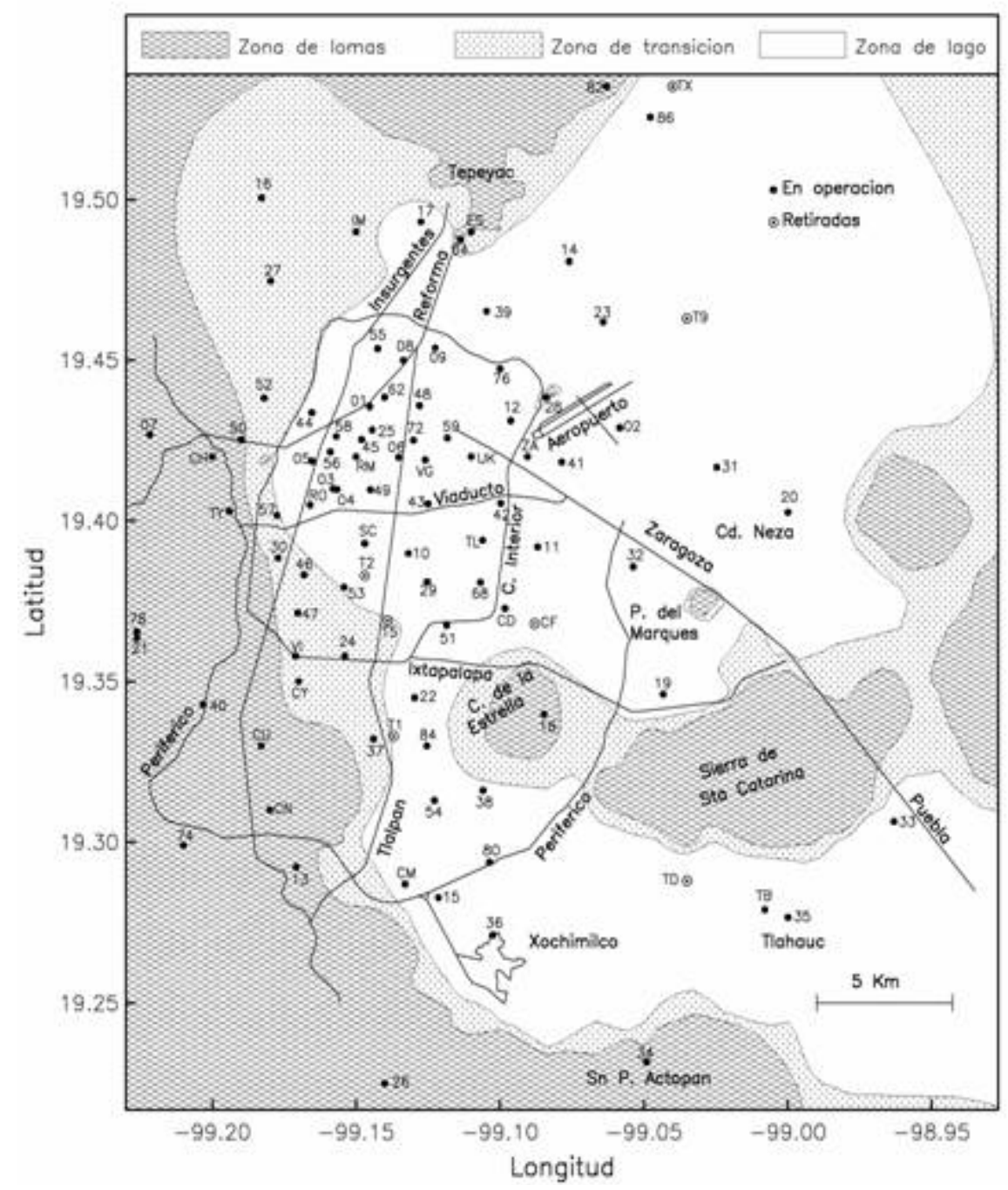

Figura 2. Estaciones de la red acelerométrica de la Ciudad de México (RACM) consideradas en el estudio (Pérez Rocha, 1998).

La más importante densificación de instrumentación sísmica en la zona metropolitana de la Ciudad de México se llevó a cabo entre 1986 y 1988, cuando más de 120 instrumentos fueron instalados, alrededor de 90 de éstos ubicados en campo libre (figura 2). La mayoría de los 
instrumentos se colocaron en la zona de lago, debido a que en ésta se han presentado los mayores daños y colapsos durante sismos de importancia (1957,1979 y 1985). La red de instrumentación existente a la fecha ha permitido realizar una gran diversidad de estudios, entre ellos algunos relacionados con la construcción de mapas de peligro y de vulnerabilidad sísmica, entre los cuales destacan los siguientes.

Gómez-Bernal et al. (1991) presentaron contornos de demandas ductilidad y de intensidad de Arias para algunas áreas de la zona metropolitana e la Ciudad de México, basándose en un modelo elastoplástico perfecto y considerando periodos entre 1.5 y $1.8 \mathrm{seg}$. Para los análisis los autores emplearon los registros del sismo del 25 de abril de 1989, realizando el escalamiento de ellos mediante la afectación de un factor $(f)$ basado en la intensidad de Arias (ec. 1).

$$
\text { fescala }=\sqrt{\frac{I_{\text {Max85 }}}{I_{M a \times 89}}}
$$

donde $I_{\text {Máx85 }}$ es la intensidad de Arias máxima registrada durante el sismo del 19 de septiembre 1985 e $I_{\text {Máx89 }}$ es la intensidad de Arias máxima registrada durante el sismo del 25 de abril 1989. Dado que el sismo de 1985 únicamente se registró en 11 estaciones acelerométricas, lo autores realizaron extrapolaciones para $I_{\text {Máx85 }}$ en varias estaciones consideradas en su estudio, tomando en consideración la estación más cercana de registro, así como las condiciones y tipo de suelo.

Pérez-Rocha (1998) presentó mapas de peligro basados en aceleraciones espectrales promedio $(\mathrm{S} a / g)$ considerando periodos estructurales de $\mathrm{T}=1 \pm 0.25 \mathrm{~s}$ y $\mathrm{T}=2 \pm 0.5 \mathrm{~s}$. En este estudio, el autor considera cuatro diferentes escenarios sísmicos potencialmente dañinos para la Ciudad de México, dentro de los cuales figuran los siguientes: (a) sismo de subducción con origen en las costas del estado de Michoacán con magnitud $\mathrm{M}_{\mathrm{s}}=8.1$ (escenario similar al del sismo del 19 de septiembre de 1985), (b) sismo de subducción con origen en las costas del estado de Guerrero con magnitud $\mathrm{M}_{\mathrm{s}}=7.7$ (escenario similar al del sismo del 28 de julio de 1957), (c) sismo de subducción con origen en las costas del estado de Guerrero con magnitud $\mathrm{M}_{\mathrm{s}}=8.2$, éste es el escenario más desfavorable estimado por los especialistas con origen en la brecha de Guerrero y, (d) sismo con mecanismo de falla normal ocurrido a una distancia de $80 \mathrm{~km}$ de la Ciudad de México. El estudio realizado por Pérez-Rocha (1998) se basa en el escalamiento de la fuente sísmica empleando para esto el modelo $\omega^{2}$, así como el cálculo y empleo de funciones de transferencia promedio obtenidas a partir de la información recabada en la red acelerométrica de la Ciudad de México (RACM) entre 1988 y 1997 (figura 2). El autor emplea la teoría de vibraciones aleatorias para la definición de espectros de respuesta elásticos en pseudoaceleración y desarrolla un modelo Bayesiano de interpolación espacial para la definición de los contornos de pseudoaceleración espectral.

Reinoso y Ordaz (1999) presentan cocientes espectrales, con la finalidad de cuantificar las amplificaciones relativas para algunos sitios representativos de la zona de lago. Asimismo, presentan mapas de igual amplificación para diferentes periodos dominantes del suelo $(\mathrm{T}=1.5 \mathrm{~s}$, $\mathrm{T}=2 \mathrm{~s}, \mathrm{~T}=3 \mathrm{~s}$ y $\mathrm{T}=5 \mathrm{~s}$ ) empleando una metodología similar a la presentada en Pérez-Rocha (1998). 
Huerta y Reinoso (2002) presentan mapas de energía elástica de entrada $\left(\mathrm{E}_{\mathrm{I}}\right)$ para el sismo del 25 de abril de $1989\left(\mathrm{M}_{\mathrm{s}}=6.9\right)$ para sistemas con diferentes periodos estructurales $\left(\mathrm{T}_{\mathrm{E}}=2 \mathrm{~s}\right.$, $\left.\mathrm{T}_{\mathrm{E}}=3 \mathrm{~s}, \mathrm{~T}_{\mathrm{E}}=4 \mathrm{~s}, \mathrm{~T}_{\mathrm{E}}=5 \mathrm{~s}\right)$. Asimismo presentan mapas de energía histerética normalizada $\left(N E_{\mathrm{H}}\right)$ considerado sistemas elastoplásticos perfectos con $\mu=4$ para el mismo escenario sísmico y los mismos periodos estructurales. De igual manera, presentan mapas de energía elástica de entrada ( $\left.\mathrm{E}_{\mathrm{I}}\right)$ para sistemas con periodos estructurales $\mathrm{T}_{\mathrm{E}}=2 \mathrm{~s}$ considerando los siguientes escenarios sísmicos: (a) sismo de subducción, ocurrido el 14 de septiembre de 1995 (M=6.4), (b) sismo de subducción, ocurrido el 30 de septiembre de 1999 (M=7.5) y, (c) sismo de falla normal, ocurrido el 15 de junio de $1999(\mathrm{M}=6.5)$.

Pineda y Ordaz (2004) presentan mapas de distribución de velocidad máxima del suelo de la Ciudad de México considerando los siguientes escenarios sísmicos: (a) sismo del 25 de abril de 1989 con magnitud $M=6.9$, (b) sismo del 14 de septiembre de 1995 con magnitud $M=7.4$, (c) sismo del 9 de octubre de 1995 con magnitud $M=8.0 \mathrm{y}$, (d) sismo del 19 de septiembre de 1985 con magnitud $\mathrm{M}=8.1$.

En su mayoría, los estudios mencionados para estimar el peligro o la vulnerabilidad sísmica del área metropolitana de la Ciudad de México se basan en parámetros elásticos (GómezBernal et al. 1991, Pérez-Rocha 1998, Reinoso y Ordaz 1999, Huerta y Reinoso 2002, Pineda y Ordaz 2004). Los únicos estudios que consideran parámetros con base en respuestas inelásticas son los desarrollados por Gómez-Bernal et al. (1991) y Huerta y Reinoso (2002).

Este estudio presenta mapas de isodemandas de ductilidad y desplazamiento para estructuras de concreto en la zona metropolitana de la Ciudad de México, considerando un escenario sísmico similar al sismo del 19 de septiembre de 1985. El estudio se enfoca exclusivamente a estructuras con base en marcos de concreto reforzado, debido a que durante los sismos de septiembre de 1985, dicho sistema estructural fue uno de los que resultó mayormente afectado, sufriendo daños graves o colapsos, como se observa en las tablas 1 y 2.

Tabla 1. Inventario de daños en función del sistema estructural empleado ("Fundación ICA", 1988)

\begin{tabular}{|c|c|c|c|c|c|c|c|c|}
\hline \multirow[b]{2}{*}{$\begin{array}{c}\text { SISTEMA } \\
\text { ESTRUCTURAL }\end{array}$} & \multicolumn{8}{|c|}{ NÚMERO DE NIVELES } \\
\hline & 1 a 2 & 3 a 5 & 6 a 8 & 9 a 12 & 12 o más & $\begin{array}{l}\text { Total de } \\
\text { Estructuras }\end{array}$ & $\begin{array}{l}\text { Estructuras } \\
\text { Dañadas }\end{array}$ & $\%$ \\
\hline Muros de Carga & 35,115 & 7,731 & 165 & 21 & 6 & 43,038 & 393 & 0.9 \\
\hline $\begin{array}{l}\text { Columnas y Losa } \\
\text { Reticular o Plana }\end{array}$ & 384 & 943 & 462 & 231 & 107 & 2,127 & 125 & 5.9 \\
\hline $\begin{array}{l}\text { Columnas, Trabes y } \\
\text { Losas }\end{array}$ & 1,961 & 4,764 & 966 & 265 & 82 & 8,038 & 236 & 2.9 \\
\hline Con Muros de Concreto & 24 & 60 & 23 & 14 & 31 & 155 & 3 & 1.9 \\
\hline Total de Estructuras & 37,484 & 13,498 & 1,616 & 531 & 229 & 53,358 & 757 & 1.4 \\
\hline Estructuras Dañadas & 346 & 179 & 136 & 72 & 24 & 757 & & \\
\hline$\%$ & 0.9 & 1.3 & 8.4 & 13.6 & 10.5 & 1.4 & & \\
\hline
\end{tabular}


Tabla 2. Inventario de daños acorde al año de construcción y al número de niveles de las estructuras (adaptado de "Instituto de Ingeniería", 1985).

\begin{tabular}{|c|c|c|c|c|c|c|c|c|c|}
\hline \multirow[t]{2}{*}{ ESTRUCTURACIÓN } & \multirow[t]{2}{*}{$\begin{array}{l}\text { TIPO DE } \\
\text { DAÑO }\end{array}$} & \multicolumn{3}{|c|}{$\begin{array}{c}\text { AÑO DE } \\
\text { CONSTRUCCIÓN }\end{array}$} & \multicolumn{4}{|c|}{ NÚMERO DE PISOS } & \multirow[t]{2}{*}{ TOTAL } \\
\hline & & $<<57$ & $57-76$ & $>76$ & $\leq 5$ & $6-10$ & $11-15$ & $>15$ & \\
\hline \multirow{2}{*}{ Marcos de Concreto } & Colapso & 27 & 51 & 4 & 27 & 46 & 8 & 1 & 82 \\
\hline & Grave & 16 & 23 & 6 & 10 & 22 & 6 & 1 & 45 \\
\hline \multirow{2}{*}{ Marcos de Acero } & Colapso & 7 & 2 & 0 & 4 & 3 & 1 & 1 & 9 \\
\hline & Grave & 1 & 3 & 0 & 0 & 0 & 2 & 2 & 4 \\
\hline \multirow[t]{2}{*}{ Losa Plana } & Colapso & 8 & 62 & 21 & 36 & 49 & 5 & 1 & 91 \\
\hline & Grave & 4 & 22 & 18 & 5 & 26 & 12 & 1 & 44 \\
\hline \multirow{2}{*}{ Mampostería } & Colapso & 6 & 5 & 2 & 11 & 2 & 0 & 0 & 13 \\
\hline & Grave & 9 & 13 & 1 & 22 & 1 & 0 & 0 & 23 \\
\hline \multirow[t]{2}{*}{ Otros } & Colapso & 7 & 5 & 2 & 12 & 2 & 0 & 0 & 14 \\
\hline & Grave & 0 & 4 & 2 & 2 & 4 & 0 & 0 & 6 \\
\hline \multirow[t]{2}{*}{ Total } & Colapso & 55 & 125 & 29 & 90 & 102 & 14 & 3 & 209 \\
\hline & Grave & 30 & 65 & 27 & 39 & 59 & 20 & 4 & 122 \\
\hline
\end{tabular}

La descripción de la metodología empleada en estudio se comenta con mayor detalle en las secciones siguientes.

\section{DESCRIPCIÓN DE LA METODOLOGÍA PROPUESTA PARA EVALUAR LA VULNERABILIDAD SÍSMICA DE ESTRUCTURAS EN EL DISTRITO FEDERAL}

La metodología propuesta en este estudio que se presenta en detalle en Godínez (2005) se basa en los trabajos desarrollados por Tena (1998, 2001) y Pérez Rocha (1998). En Pérez Rocha (1998) se presentan mapas de vulnerabilidad con base en contornos de aceleraciones espectrales máximas a partir del cálculo de espectros de aceleración en el Valle de México considerando diferentes escenarios sísmicos a partir de escalar la fuente sísmica, del empleo de funciones de transferencia promedio para los sitios instrumentados del valle y de la interpolación espacial de éstos con base en técnicas Bayesianas.

En Tena $(1998,2001)$ se propone una metodología de evaluación sísmica simplificada de estructuras que implica el cálculo de espectros de demandas de ductilidad, ya que son de gran utilidad para la revisión de estructuras existentes ante sismos. Los espectros de demandas de ductilidad relacionan las demandas de ductilidad de sistemas de un grado de libertad con diferentes periodos para una resistencia lateral dada, es decir, los espectros de demandas de ductilidad son espectros de isorresistencias.

Esta investigación se concentra en el estudio exclusivo de estructuras con base en marcos de concreto reforzado, ya que este sistema fue uno de los que resultaron con mayores daños durante los sismos de septiembre de 1985, como se concluye del análisis de las tablas 1 y 2 . Dado que las estructuras consideradas son de concreto reforzado, se consideró un modelo de histéresis que represente de la mejor manera posible el comportamiento de este material. 
La metodología empleada se ilustra esquemáticamente en la figura 3, y se describe con cierto detalle en las siguientes secciones.
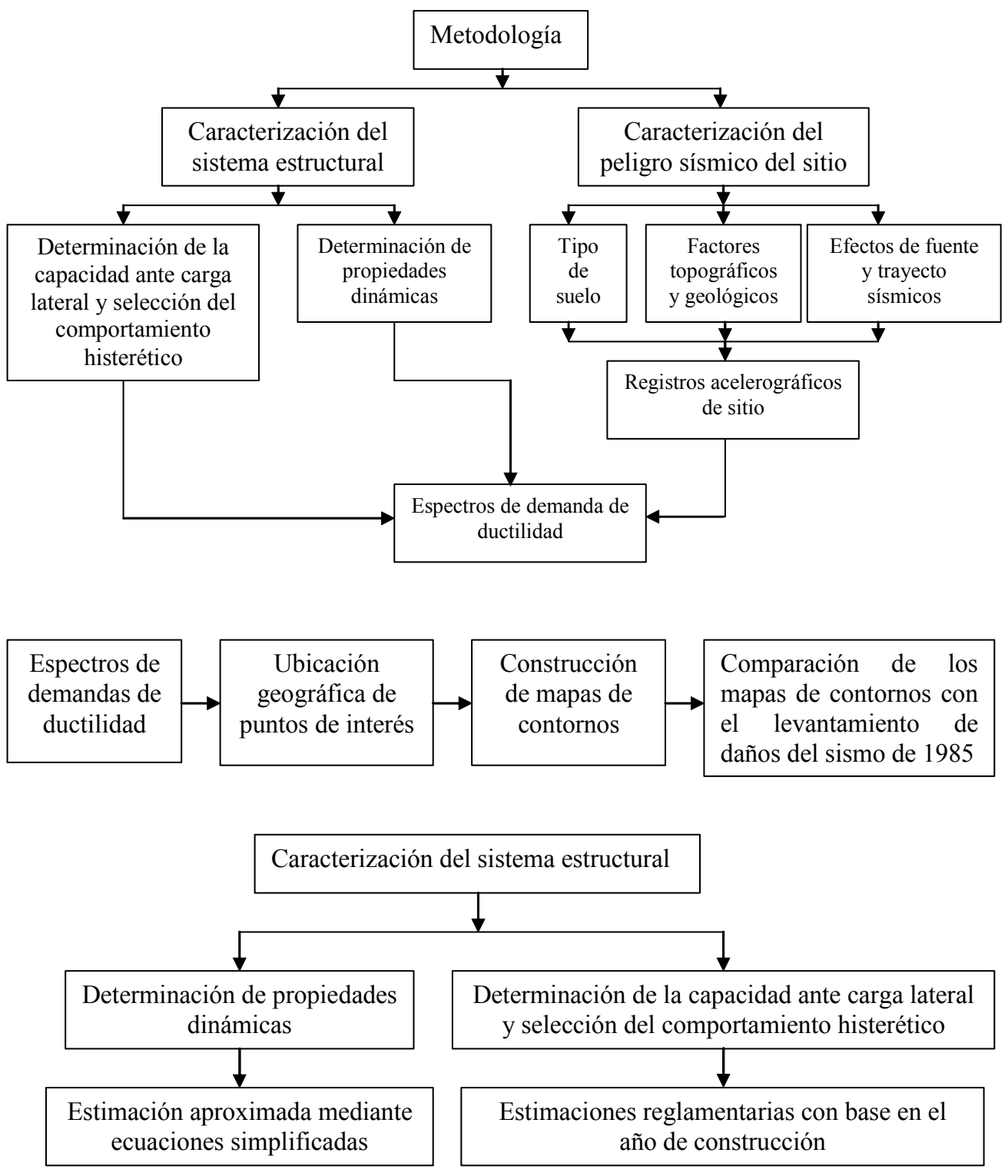

Figura 3. Metodología propuesta para la evaluación de la vulnerabilidad sísmica de estructuras existentes.

\section{CARACTERIZACIÓN DEL PELIGRO SÍSMICO}

\section{Determinación de espectros de Amplitudes de Fourier (EAF) para terreno firme}

Existe un método complementario en el cual se emplean los registros de sismos pequeños $(\mathrm{M}<6)$ y moderados $(6<\mathrm{M}<7)$ para simular el movimiento producido por sismos mayores. Lo planteado 
se apoya en la hipótesis de que la complejidad observada en los registros estará presente en aquéllos producidos por sismos de mayor magnitud originados en la misma región epicentral. Los efectos atribuidos a la fuente (discontinuidades en el área de contacto entre placas, efectos de directividad, velocidad de ruptura, particularidades de la irradiación energética, etc.) y al trayecto (principalmente la atenuación), se preservan en temblores grandes y chicos (Pérez-Rocha, 1998).

En 1988 Singh y colaboradores describieron en forma cuantitativa las amplificaciones relativas del movimiento del terreno mediante funciones de transferencia empíricas (FTE). De acuerdo con los autores, las amplificaciones relativas del movimiento (norte-sur y este-oeste) se pueden representar mediante el cociente:

$$
H_{i}(\omega)=\frac{\left|A_{i}(\omega)\right|}{\left|A_{C U}(\omega)\right|}
$$

donde $H_{i}(\omega)$ y $\left|A_{i}(\omega)\right|$ son las FTE y el EAF del movimiento en el sitio, respectivamente, mientras $\left|A_{C U}(\omega)\right|$ es el EAF del movimiento en el sitio CU (Pérez Rocha, 1998).

Para un sitio arbitrario, el EAF se especifica mediante el producto entre la FTE del sitio y el EAF que se tendría en el terreno firme ante un sismo postulado (Pérez-Rocha, 1998).

Para cada sitio de registro (92 estaciones en campo libre, figura 2), Pérez Rocha (1998) obtuvo medidas estadísticas para describir las amplificaciones dinámicas observadas en las FTE debidas a varios temblores. El autor calculó las FTE promedio (dos componentes horizontales) y los coeficientes de variación, que son relativamente pequeños (entre 0.1 y 0.3 ). El propósito de esto fue construir una base de datos de FTE para un modelo de interpolación espacial que propone el autor con el fin de estimar de una manera aceptable las FTE en sitios no instrumentados del área metropolitana de la Ciudad de México.

\section{Generación de acelerogramas sintéticos}

El procedimiento planteado para la obtención de los acelerogramas sintéticos, contempla el empleo de las FTE promedio obtenidas por Pérez Rocha hasta 2004, a partir de los registros de la red acelerométrica de la Ciudad de México y el espectro promedio de amplitudes de Fourier correspondiente a terreno firme. En este caso particular, se toma el promedio de terreno firme como el promedio observado en CU.

Las FTE se calculan con respecto al EAF promedio del terreno firme, debido a que con esto la amplificación obtenida para la mayoría de los sitios es razonablemente constante de temblor a temblor, sin importar la magnitud, la distancia epicentral o el azimut de la incidencia (Reinoso 1991, Reinoso y Ordaz 1999). 
Durante el proceso de escalamiento de la fuente sísmica, se emplearon como base (funciones de Green) los registros acelerométricos correspondientes al sismo del 25 de abril de 1989, de magnitud $\mathrm{M}=6.9$ originado en la brecha de San Marcos.

La generación de los acelerogramas sintéticos se realizó mediante un programa especializado. El procedimiento general para la obtención de los registros sintéticos es el siguiente:

1. Conociendo el registro acelerográfico de terreno firme (Ciudad Universitaria) del sismo empleado como función de Green, se calcula el espectro de amplitudes de Fourier promedio para terreno firme $\left(\mathrm{EAF}_{\mathrm{TF}}\right)$ correspondiente.

2. Selección de la función de transferencia promedio correspondiente a terreno firme $\left(\mathrm{FTE}_{\mathrm{TF}}\right)$.

3. Seleccionar una función de transferencia correspondiente al sitio de interés (FTE $\mathrm{FI}_{\mathrm{SI}}$, calculada con respecto al EAF promedio del terreno firme $\left(\mathrm{EAF}_{\mathrm{TF}}\right)$.

4. Calcular el espectro de amplitudes de Fourier para el sitio de interés $\left(\mathrm{EAF}_{\mathrm{SI}}\right)$, mediante el producto del espectro de amplitudes de Fourier en terreno firme $\left(\mathrm{EAF}_{\mathrm{TF}}\right)$ y la FTE promedio para el sitio de interés $\left(\mathrm{FTE}_{\mathrm{SI}}\right)$, dividida por la función de transferencia promedio correspondiente a terreno firme $\left(\mathrm{FTE}_{\mathrm{TF}}\right)$.

5. Calcular el espectro de amplitudes de Fourier para el sitio de interés para la magnitud del sismo de interés $\left(\mathrm{EAF}_{\mathrm{SIMS}}\right)$, a partir del $\mathrm{EAF}_{\mathrm{SI}}$ y de escalar la fuente sísmica. Calcular el espectro de pseudoaceleración asociado al sismo escalado ( $\mathrm{Sa}_{\text {SIMS }}$ ) a partir del $\mathrm{EAF}_{\text {SIMS. }}$

6. Calcular el acelerograma sintético (AS) a partir del $\mathrm{EAF}_{\text {SIMS }}$ y de utilizar fases aleatorias de ruido blanco gaussiano. Se considera también la duración de la fase intensa observada, o en caso de no contar con esta información, se estima con base en la observada en otros sitios análogos. Normalmente se generan varios acelerogramas sintéticos y se selecciona aquél cuyos espectros de amplitud de Fourier $\left(\mathrm{EAF}_{\mathrm{AS}}\right)$ y de pseudoaceleraciones $\left(\mathrm{Sa}_{\mathrm{AS}}\right)$ se aproximen razonablemente con los utilizados como referencia $\left(\mathrm{EAF}_{\text {SIMS }} \mathrm{y}\right.$ SasIMS $)$.

En la figura 4 se ilustra la fase final del escalamiento de la fuente sísmica para la obtención de un acelerograma sintético. En esta figura se muestran los espectros de amplitudes de Fourier, los espectros de respuesta elásticos y el acelerograma sintético resultante. Los espectros marcados con el número uno (amarillo), corresponden a la función objetivo (espectros de referencia, Sasims y $\mathrm{EAF}_{\text {SIMS }}$ ), los marcados con el número dos (azul), corresponden al acelerograma sintético generado $\left(\mathrm{Sa}_{\mathrm{AS}}\right.$ y $\left.\mathrm{EAF}_{\mathrm{AS}}\right)$, y los espectros marcados con el número tres (rojo, SasI y EAF $F_{\text {SI }}$ ) son los correspondientes al sismo "semilla".

\section{CARACTERIZACIÓN DEL SISTEMA ESTRUCTURAL}

Dado que la mayoría de los sistemas estructurales con base en marcos de concreto reforzado que sufrieron daños severos o colapsos contaban con alturas medias, es decir, oscilaban entre los 
cinco y los quince niveles (diseñados conforme a los reglamentos vigentes previos a los sismos de septiembre de 1985, tabla 2), se realizó un estudio de su vulnerabilidad considerando varios factores. Para dicho estudio es necesario realizar una estimación de las propiedades dinámicas de los sistemas en cuestión, estimar su resistencia ante cargas laterales y definir adecuadamente los modelos de comportamiento histerético a emplear en cada caso de estudio, así como definir los registros acelerométricos acorde a la zona de estudio.

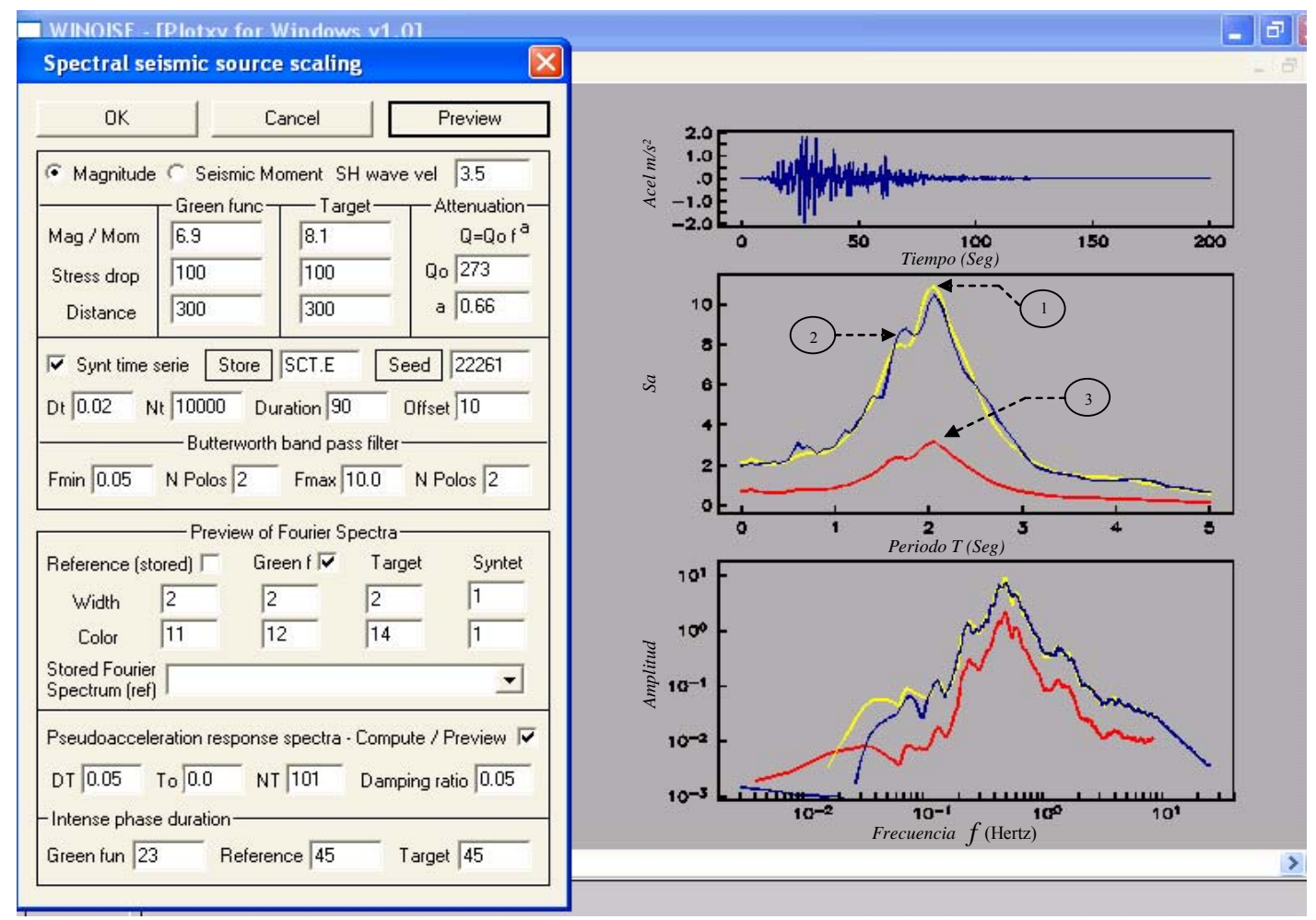

Figura 4. Fase final en el proceso de generación de un acelerograma sintético

\section{Determinación aproximada de periodos naturales de vibración}

Para la determinación de los periodos de vibración de edificios de concreto reforzado ubicados en la Ciudad de México, se emplearon las expresiones propuestas por Murià y González (1995), las cuales se muestran en la tabla 3. Estas expresiones están basadas en un estudio realizado con la finalidad de determinar las propiedades dinámicas de edificios en la Ciudad de México a partir de mediciones de vibración ambiental, vibración forzada y registros sísmicos. Cabe destacar que las expresiones de la tabla 3 son aplicables a estructuras que cuenten con un número de pisos no mayor a 20 .

Empleando las expresiones de la tabla 3, se calcularon los periodos fundamentales de vibración para estructuras que van desde uno hasta 20 niveles, los resultados se muestran en la tabla 4. 
Tabla 3. Expresiones para el cálculo aproximado de periodos naturales de vibración para estructuras de concreto reforzado en la Ciudad de México (adaptada de Murià y González, 1995)

\begin{tabular}{|c|c|c|}
\hline SISTEMA ESTRUCTURAL & " SUELO FIRME & SUELO BLANDO \\
\hline Marcos & $T=0.100 N$ & $T=0.126 N$ \\
\hline
\end{tabular}

Los periodos mostrados en la tabla 4 corresponden a los límites superior e inferior de cada intervalo considerado respecto al número de niveles de la estructura, el cual está acorde con el reporte de daños realizado por el Instituto de Ingeniería de la UNAM en 1986 (tabla 2), donde se presentan de manera detallada el número de estructuras que sufrieron diferentes niveles de daño.

Tabla 4. Periodos fundamentales de vibración para sistemas con base en marcos de concreto

\begin{tabular}{|c|c|c||}
\hline MARCOS DE CONCRETO & TERRENO FIRME & TERRENO BLANDO \\
\hline Número de Niveles & Periodos (s) & Periodo (s) \\
\hline$<5$ & $0.1-0.5$ & $0.13-0.63$ \\
\hline 6 a 10 & $0.6-1.0$ & $0.76-1.26$ \\
\hline 11 a 15 & $1.1-1.5$ & $1.39-1.89$ \\
\hline 16 a 20 & $1.6-2.0$ & $2.02-2.52$ \\
\hline
\end{tabular}

\section{Determinación de la capacidad del sistema estructural ante carga lateral}

Las resistencias nominales ante carga lateral de las estructuras con base en marcos de concreto reforzado existentes en el valle de México se estimaron conforme a las mínimas asociadas a un diseño conforme a los reglamentos de construcción que estuvieron vigentes antes de los sismos de septiembre de 1985: RCDF-42, RCDF-57, RCDF-66 y RCDF-76. En la tabla 5 se presentan los coeficientes sísmicos $(c)$ correspondientes a los espectros de diseño elásticos que se estipulan en cada uno de los reglamentos en estudio, asociadas a los periodos naturales calculados. Para los RCDF-1957 y RCDF-1966, las estructuras tipo B1 hacen referencia a aquéllas con muros no estructurales ligados al sistema principal y que contribuyen a aumentar la rigidez del sistema, en tanto que las estructuras B2 hacen referencia a aquéllas donde los muros no estructurales se encuentran desligados adecuadamente del sistema estructural ("Fundación ICA", 1988).

Con fines ilustrativos, en las figuras 5, 6 y 7 se muestran los espectros de diseño elásticos correspondientes a las zonas I, II y III respectivamente, de los cuales se observa la evolución que tuvieron los reglamentos de diseño sísmico para el Distrito Federal desde 1942 hasta 1976 para estructuras del grupo B. En efecto, fue hasta 1976 que se contempló como tal un espectro de diseño sísmico; para los años precedentes la variación de la resistencia era independiente del periodo natural de vibración de la estructura, ya que dependía únicamente de la zona considerada (figura 5, tabla 5).

El RCDF-76 fue el primer reglamento mexicano basado en criterios de diseño por resistencia última; los reglamentos precedentes estaban basados en diseño por esfuerzos permisibles. 
Vulnerabilidad de estructuras con base en marcos de concreto reforzado en el Valle de México ante un escenario...

Tabla 5. Coeficientes sísmicos $c=\mathrm{V} / \mathrm{W}$ para sistemas con base en marcos de concreto, acorde a los reglamentos de construcción en estudio.

\begin{tabular}{|c|c|c|c|c|c|c|c|}
\hline \multicolumn{8}{|c|}{ ZZona I (zona de terreno firme) } \\
\hline Número de Niveles & Periodo (s) & RCDF-42 & $\begin{array}{c}\text { RCDF-57 } \\
\text { (B1) }\end{array}$ & $\begin{array}{l}\text { RCDF-57 } \\
\text { (B2) }\end{array}$ & $\begin{array}{c}\text { RCDF-66 } \\
\text { (B1) }\end{array}$ & $\begin{array}{c}\text { RCDF-66 } \\
\text { (B2) }\end{array}$ & RCDF-76 \\
\hline$\overline{<5}$ & $0.0-0.5$ & 0.025 & 0.05 & 0.07 & 0.04 & 0.08 & $0.03-0.16$ \\
\hline 6 a 10 & $0.6-1.0$ & 0.025 & 0.05 & 0.07 & 0.04 & 0.08 & $0.16-0.14$ \\
\hline 11 a 15 & $1.1-1.5$ & 0.025 & 0.05 & 0.07 & 0.04 & 0.08 & $0.14-0.12$ \\
\hline 16 a 20 & $1.6-2.0$ & 0.025 & 0.05 & 0.07 & 0.04 & 0.08 & $0.11-0.10$ \\
\hline \multicolumn{8}{|c|}{ Zona II (zona de transición) } \\
\hline Número de Niveles & Periodo (s) & RCDF-42 & $\begin{array}{c}\text { RCDF-57 } \\
\text { (B1) }\end{array}$ & $\begin{array}{c}\text { RCDF-57 } \\
\text { (B2) }\end{array}$ & $\begin{array}{c}\text { RCDF-66 } \\
\text { (B1) }\end{array}$ & $\begin{array}{c}\text { RCDF-66 } \\
\text { (B2) }\end{array}$ & RCDF-76 \\
\hline$<5$ & $0.0-0.5$ & 0.025 & 0.06 & 0.09 & 0.06 & 0.08 & $0.045-0.2$ \\
\hline 6 a 10 & $0.6-1.0$ & 0.025 & 0.06 & 0.09 & 0.06 & 0.08 & 0.2 \\
\hline 11 a 15 & $1.1-1.5$ & 0.025 & 0.06 & 0.09 & 0.06 & 0.08 & 0.2 \\
\hline 16 a 20 & $1.6-2.0$ & 0.025 & 0.06 & 0.09 & 0.06 & 0.08 & 0.2 \\
\hline \multicolumn{8}{|c|}{ Zona III (zona de lago) } \\
\hline Número de Niveles & Periodo (s) & RCDF-42 & $\begin{array}{c}\text { RCDF-57 } \\
\text { (B1) }\end{array}$ & $\begin{array}{c}\text { RCDF-57 } \\
\text { (B2) }\end{array}$ & $\begin{array}{c}\text { RCDF-66 } \\
\text { (B1) }\end{array}$ & $\begin{array}{c}\text { RCDF-66 } \\
\text { (B2) }\end{array}$ & RCDF-76 \\
\hline$<5$ & $0.0-0.63$ & 0.025 & 0.07 & 0.1 & 0.06 & 0.08 & $0.06-0.20$ \\
\hline 6 a 10 & $0.76-1.26$ & 0.025 & 0.07 & 0.1 & 0.06 & 0.08 & $0.23-0.24$ \\
\hline 11 a 15 & $1.39-1.89$ & 0.025 & 0.07 & 0.1 & 0.06 & 0.08 & 0.24 \\
\hline 16 a 20 & $2.02-2.52$ & 0.025 & 0.07 & 0.1 & 0.06 & 0.08 & 0.24 \\
\hline
\end{tabular}

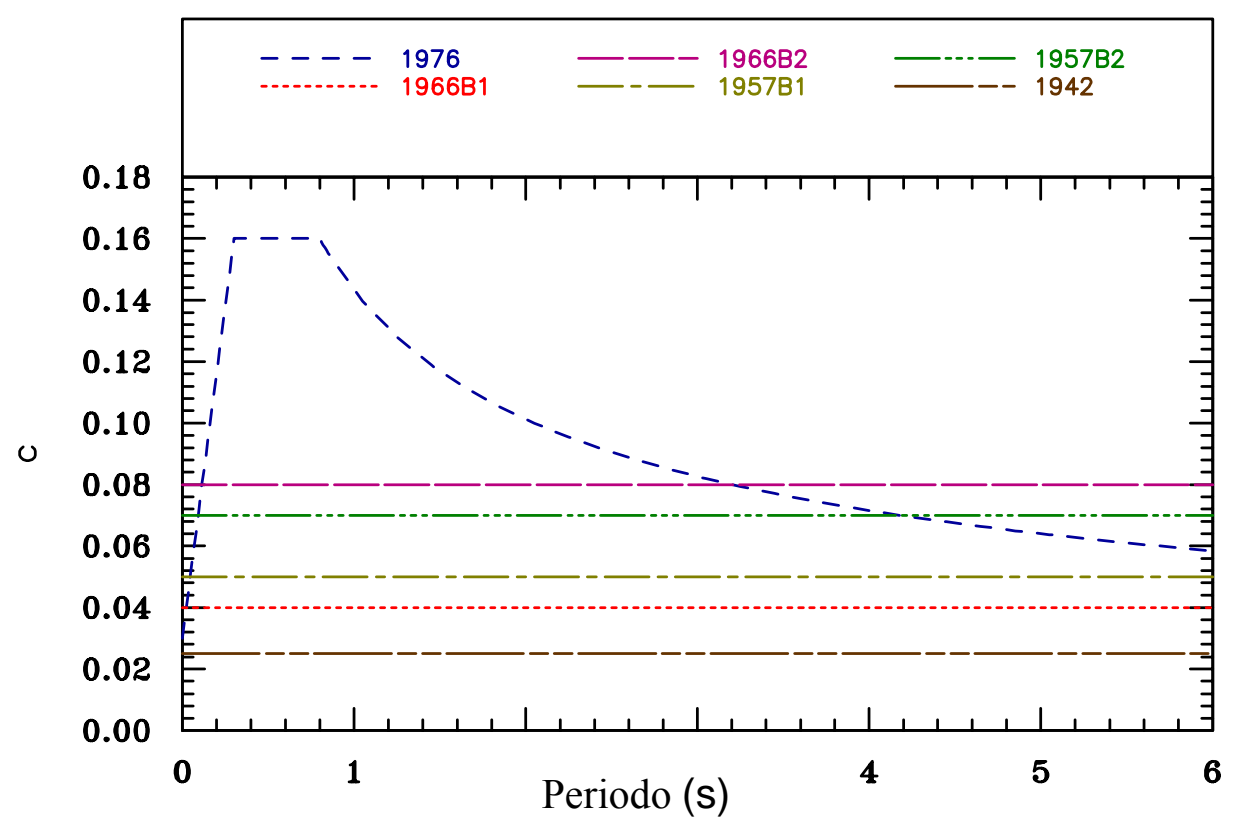

Figura 5. Evolución de los espectros de diseño elásticos de 1942 a 1976 en zona I. 


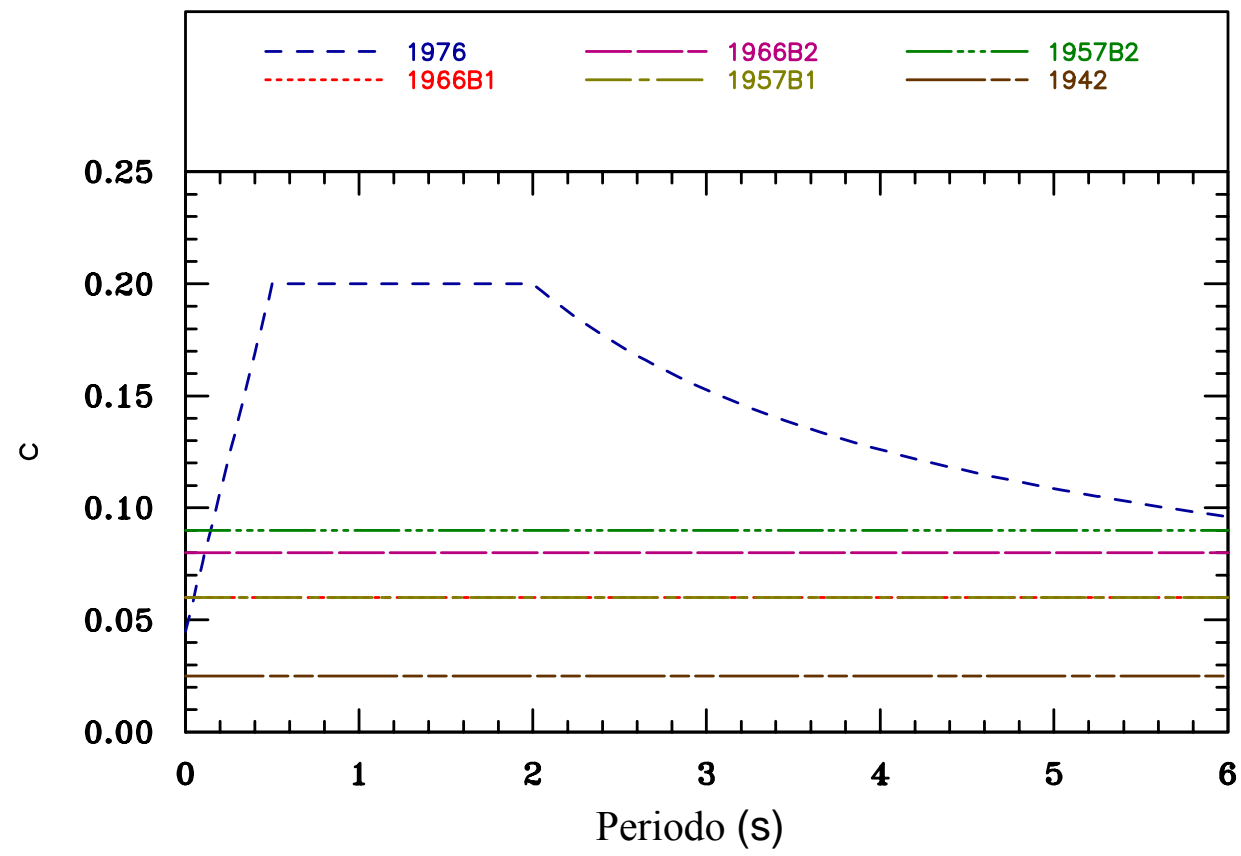

Figura 6. Evolución de los espectros de diseño elástıcos de 1942 a 1976 en zona II.

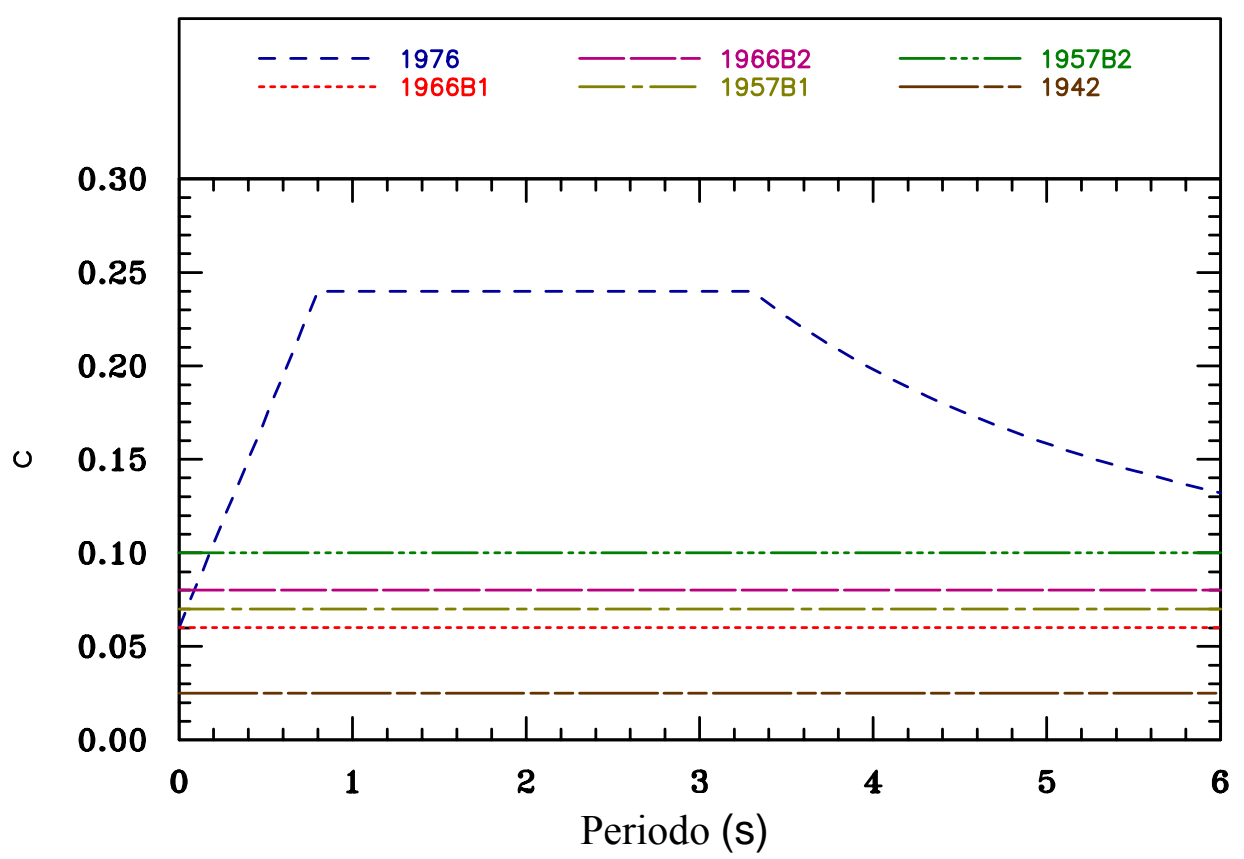

Figura 7. Evolución de los espectros de diseño elásticos de 1942 a 1976 en zona III.

En el RCDF-76 se introdujeron por vez primera los factores de comportamiento sísmico $\mathrm{Q}$ y de reducción de fuerzas sísmicas Q'. Para el diseño de marcos de concreto se permitieron valores de $\mathrm{Q}=4$ y $\mathrm{Q}=6$. Por tanto, se requirió del cálculo de los valores de los coeficientes sísmicos reducidos por ductilidad ( $c / \mathrm{Q}^{\prime}$, debido a comportamiento inelástico), los cuales se presentan en la tabla 6 . 
Los valores marcados con asterisco en la tabla 6 son menores a la aceleración del terreno estipuladas para cada una de las distintas zonas. De acuerdo con el artículo 240 del Reglamento de Construcciones para el Distrito Federal, en la parte respectiva a diseño por sismo, ningún valor reducido por ductilidad podrá ser menor a la aceleración del terreno $\left(\mathrm{a}_{\mathrm{o}}\right)$, por lo que para la zona I, el valor por el que se sustituyen todos los valores marcados con asterisco es igual a 0.03 , para zona II el valor es 0.045, y para zona III dicho valor es 0.06 . Dichos valores fueron los que se emplearon en los cálculos de los espectros de demanda de ductilidad y desplazamiento.

Tabla 6. Coeficientes sísmicos reducidos por Q' para marcos de concreto diseñados con base en el RCDF-76.

\begin{tabular}{|c|c|c|c|c|}
\hline \multicolumn{5}{|c|}{ Terreno firme (zona I) } \\
\hline \multirow[t]{2}{*}{ Número de Niveles } & \multirow[t]{2}{*}{ Periodos (s) } & \multirow[t]{2}{*}{$c(1976)$} & \multicolumn{2}{|c|}{ Coeficiente sísmico reducido (c/Q') } \\
\hline & & & $\mathrm{Q}=4$ & $\mathrm{Q}=6$ \\
\hline$<5$ & 0.5 & 0.16 & 0.040 & $0.027 *$ \\
\hline 6 a 10 & $0.6-1.0$ & $0.16-0.1431$ & $0.04-0.036$ & $0.024 *$ \\
\hline 11 a 15 & $1.1-1.5$ & $0.1364-0.1168$ & $0.036-0.029 *$ & $0.019 *$ \\
\hline 16 a 20 & $1.6-2.0$ & $0.1131-0.1012$ & $0.025 *$ & $0.017 *$ \\
\hline \multicolumn{5}{|c|}{ Terreno de transición (zona II) } \\
\hline \multirow[t]{2}{*}{ Número de Niveles } & \multirow[t]{2}{*}{ Periodos (s) } & \multirow[t]{2}{*}{ c (1976) } & \multicolumn{2}{|c|}{ Coeficiente sísmico reducido (c/Q') } \\
\hline & & & $\mathrm{Q}=4$ & $\mathrm{Q}=6$ \\
\hline$<5$ & $0.0-0.63$ & $0.045-0.20$ & 0.050 & $0.033 *$ \\
\hline 6 a 10 & $0.756-1.26$ & 0.20 & 0.050 & $0.033 *$ \\
\hline 11 a 15 & $1.386-1.89$ & 0.20 & 0.050 & $0.033 *$ \\
\hline 16 a 20 & $2.01-2.52$ & 0.20 & 0.050 & $0.033 *$ \\
\hline \multicolumn{5}{|c|}{ Terreno blando (zona de lago o zona III) } \\
\hline \multirow[t]{2}{*}{ Número de Niveles } & \multirow[t]{2}{*}{ Periodos (s) } & \multirow[t]{2}{*}{$c(1976)$} & \multicolumn{2}{|c|}{ Coeficiente sísmico reducido (c/Q') } \\
\hline & & & $\mathrm{Q}=4$ & $Q=6$ \\
\hline$<5$ & $0.0-0.63$ & $0.06-0.2018$ & 0.0600 & 0.0409 * \\
\hline 6 a 10 & $0.756-1.26$ & $0.2301-0.24$ & 0.0600 & 0.0400 * \\
\hline 11 a 15 & $1.386-1.89$ & 0.24 & 0.0600 & 0.0400 * \\
\hline 16 a 20 & $2.016-2.52$ & 0.24 & 0.0600 & $0.0400 *$ \\
\hline
\end{tabular}

Se valoró también la sobrerresistencia asociada a estos sistemas estructurales, considerando para los análisis un factor de sobrerresistencia igual a dos $\left(\mathrm{F}_{\mathrm{SR}}=2\right)$. Los valores de los coeficientes sísmicos al considerar este efecto se muestran en la tabla 7. El factor de sobrerresistencia empleado es considerado representativo acorde lo reportado en investigaciones realizadas en México (por ejemplo, Correa 2005); además, es importante destacar que en el nuevo RCDF-2004 dentro de la parte correspondiente a diseño por sismo, se propone el empleo de un factor de sobrerresistencia de dos $\left(\mathrm{F}_{\mathrm{SR}}=2\right)$ para el diseño de estructuras con periodos naturales de vibración mayores de 0.5 segundos. 
Tabla 7. Resistencias ante carga lateral considerando efectos de sobrerresistencia para sistemas con base en marcos de concreto, acorde a los reglamentos de construcción en estudio.

\begin{tabular}{|c|c|c|c|c|c|c|c|}
\hline \multicolumn{8}{|c|}{ Zona I (zona de terreno firme) } \\
\hline Número de Niveles & Periodo (s) & RCDF-42 & $\begin{array}{c}\text { RCDF-57 } \\
\text { (B1) }\end{array}$ & $\begin{array}{c}\text { RCDF-57 } \\
\text { (B2) }\end{array}$ & $\begin{array}{c}\text { RCDF-66 } \\
\text { (B1) }\end{array}$ & $\begin{array}{c}\text { RCDF-66 } \\
\text { (B2) }\end{array}$ & $\begin{array}{c}\text { RCDF-76 } \\
Q=4\end{array}$ \\
\hline$<5$ & $0.0-0.5$ & 0.05 & 0.1 & 0.14 & 0.08 & 0.16 & 0.08 \\
\hline 6 a 10 & $0.6-1.0$ & 0.05 & 0.1 & 0.14 & 0.08 & 0.16 & $0.08-0.072$ \\
\hline 11 a 15 & $1.1-1.5$ & 0.05 & 0.1 & 0.14 & 0.08 & 0.16 & $0.072-0.06$ \\
\hline 16 a 20 & $1.6-2.0$ & 0.05 & 0.1 & 0.14 & 0.08 & 0.16 & 0.06 \\
\hline \multicolumn{8}{|c|}{ Zona II (zona de transición) } \\
\hline Número de Niveles & Periodo (s) & RCDF-42 & $\begin{array}{c}\text { RCDF-57 } \\
\text { (B1) }\end{array}$ & $\begin{array}{l}\text { RCDF-57 } \\
\text { (B2) }\end{array}$ & $\begin{array}{c}\text { RCDF-66 } \\
\text { (B1) }\end{array}$ & $\begin{array}{c}\text { RCDF-66 } \\
\text { (B2) }\end{array}$ & $\begin{array}{c}\text { RCDF-76 } \\
Q=4\end{array}$ \\
\hline$<5$ & $0.0-0.5$ & 0.05 & 0.12 & 0.18 & 0.12 & 0.16 & 0.10 \\
\hline 6 a 10 & $0.6-1.0$ & 0.05 & 0.12 & 0.18 & 0.12 & 0.16 & 0.10 \\
\hline 11 a 15 & $1.1-1.5$ & 0.05 & 0.12 & 0.18 & 0.12 & 0.16 & 0.10 \\
\hline 16 a 20 & $1.6-2.0$ & 0.05 & 0.12 & 0.18 & 0.12 & 0.16 & 0.10 \\
\hline \multicolumn{8}{|c|}{ Zona III (zona de lago) } \\
\hline Número de Niveles & Periodo (s) & RCDF-42 & $\begin{array}{c}\text { RCDF-57 } \\
\text { (B1) }\end{array}$ & $\begin{array}{l}\text { RCDF-57 } \\
\text { (B2) }\end{array}$ & $\begin{array}{c}\text { RCDF-66 } \\
\text { (B1) }\end{array}$ & $\begin{array}{c}\text { RCDF-66 } \\
\text { (B2) }\end{array}$ & $\begin{array}{c}\text { RCDF-76 } \\
Q=4\end{array}$ \\
\hline$<5$ & $0.0-0.63$ & 0.05 & 0.14 & 0.2 & 0.12 & 0.16 & 0.12 \\
\hline 6 a 10 & $0.76-1.26$ & 0.05 & 0.14 & 0.2 & 0.12 & 0.16 & 0.12 \\
\hline 11 a 15 & $1.39-1.89$ & 0.05 & 0.14 & 0.2 & 0.12 & 0.16 & 0.12 \\
\hline 16 a 20 & $2.02-2.52$ & 0.05 & 0.14 & 0.2 & 0.12 & 0.16 & 0.12 \\
\hline
\end{tabular}

\section{Modelo de comportamiento histerético considerado}

El modelo de histéresis empleado en el cálculo de los espectros de demandas de ductilidad y desplazamiento es una ligera variación del modelo de Takeda, en el cual la curva primaria fue modificada a una de tipo bilineal; la modificación fue desarrollada por Otani (Saiidi y Sozen 1979).

Dado que los detallados de armado y configuraciones estructurales en las estructuras de concreto reforzado suelen presentar variaciones importantes, a partir de 1976 el reglamento de construcciones del Distrito Federal contempló dos tipos de diseños, basándose en la ductilidad que puede desarrollar dichos sistemas estructurales, a través de la asignación de valores diferentes del factor de comportamiento sísmico, diferenciando con esto un diseño dúctil de uno no tan dúctil. Para los reglamentos anteriores (1942 a 1946), el nivel de detallado utilizado en marcos de concreto podía considerarse como no dúctil. Por lo anterior, se requirió del empleo de dos variantes en el modelo de Takeda que representaran cada uno de los comportamientos antes descritos: una primera variante que representara a estructuras no dúctiles y una segundo a estructuras dúctiles. En cada uno de los casos anteriores se realizó una valoración de los parámetros de Takeda $\mathrm{B}_{0} \mathrm{y}_{1}$, siendo $\mathrm{B}_{0}$ un factor de degradación de rigidez para ciclos grandes y $\mathrm{B}_{1}$ un factor de degradación de rigidez para ciclos pequeños. El efecto de los parámetros $\mathrm{B}_{0} \mathrm{y}$ $\mathrm{B}_{1}$ en las características de los lazos de histéresis se ilustra empleando una señal senoidal de amplitud máxima igual a $0.2 \mathrm{~g}$ y duración de $80 \mathrm{seg}$. (figura 8 ). 
Con lo anterior se obtuvieron modelos de histéresis acorde a los requerimientos marcados; los modelos considerados para cada caso se muestran en las figura 8, empleando para el caso de estructuras dúctiles un valor de $\mathrm{B}_{0}=0$ y $\mathrm{B}_{1}=1$, lo cual implica que no existe degradación de rigidez importante en el ciclo histerético y para el caso de estructuras no dúctiles los parámetros $\mathrm{B}_{0}$ y $\mathrm{B}_{1}$ tienen valores de 0.1 y 0.9 respectivamente.
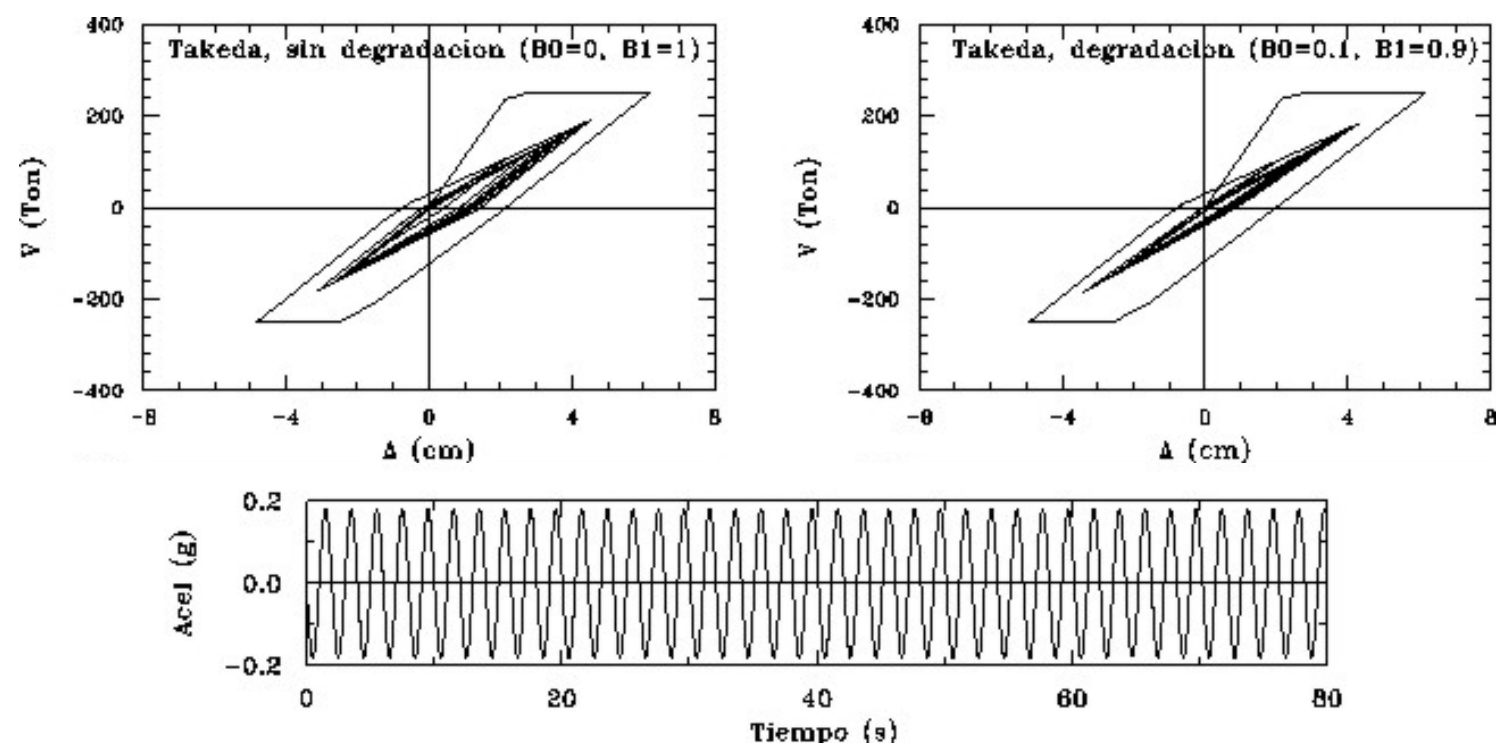

Figura 8. Modelos de histéresis de Takeda considerados en el cálculo de los espectros de demanda de ductilidad y desplazamiento. Se ilustra la respuesta obtenida para un sistema de un grado de libertad a partir de un pulso senoidal de amplitud $0.2 \mathrm{~g}$, periodo de 2.0 segundos y duración de 80 segundos.

\section{MAPAS DE ISODEMANDAS DE DUCTILIDAD Y DESPLAZAMIENTO}

Contando ya con los elementos necesarios para caracterizar al sistema estructural (propiedades dinámicas, resistencias laterales y modelo de histéresis de Takeda) y a los movimientos del terreno, se procedió al cálculo de los espectros de demanda de ductilidad y desplazamiento en dos componentes ortogonales para cada uno de los registros acelerométricos en estudio y para cada uno de los reglamentos considerados.

Dentro de los cálculos realizados para la obtención de los espectros de demanda, se contemplan dos casos de estudio, el primero, en el cual se considera la resistencia lateral nominal, $\mathrm{y}$ un segundo en que se considera un factor de sobrerresistencia de dos $\left(\mathrm{F}_{\mathrm{SR}}=2\right)$, esto con el fin de observar de manera cuantitativa el efecto de la sobrerresistencia en los parámetros de estudio.

Para el caso en que se consideran las resistencias estipuladas en los reglamentos de 1942, 1957 y 1966 en el cálculo de los espectros de demandas, dado que en los reglamentos en cuestión no existían formalmente los requerimientos de detallado para garantizar un comportamiento dúctil, se empleó un modelo de histéresis con mayor degradación que representara más fielmente a estructuras no dúctiles, por lo que en el modelo de Takeda se consideró que $\mathrm{B}_{0}=0.1$ y $\mathrm{B}_{1}=0.9$. 
Para el caso en que se consideran las resistencias estipuladas en el reglamento de 1976 para el cálculo de los espectros de demandas, se empleó un modelo de histéresis correspondiente a estructuras con menor degradación de rigidez (fig. 8), para este caso se consideraron valores de $\mathrm{B}_{0}=0$ y $\mathrm{B}_{1}=1$.

En el estudio realizado se consideró un intervalo de periodos estructurales de 0 a 5 segundos para el cálculo de espectros de demandas de ductilidad. Debido a que las estructuras con base en marcos de concreto que sufrieron mayores daños y colapsos durante el sismo de septiembre de 1985 contaban con un número de niveles que variaba entre cinco y quince (tabla 2 ), se definió un intervalo de periodos de interés $(0.5 \leq T \leq 2.0)$ para la construcción de los mapas de isodemandas.

Se presentan mapas correspondientes a periodos estructurales de $0.5,1.0,1.5$ y 2.0 segundos, considerando resistencias nominales (tablas 5 y 6) y tomando en cuenta efectos de sobrerresistencia (tabla 7). En todos los casos se consideró un factor de sobrerresistencia de dos $\left(\mathrm{F}_{\mathrm{SR}}=2\right)$.

Para cada reglamento en estudio (RCDF-42, RCDF-57(B1), RCDF-57(B2), RCDF66(B1), RCDF-66(B2), RCDF-76(Q=4), RCDF-76(Q=6)) se calcularon 32 mapas de isodemandas, considerando cada uno de los periodos estructurales de interés y dos componentes ortogonales del movimiento del terreno. En total se construyeron 224 mapas de vulnerabilidad, de los cuales 112 corresponden a isodemandas de ductilidad y 112 a isodemandas de desplazamiento inelástico para marcos de concreto reforzado, los cuales se presentan en Godínez (2005).

Debido a la insuficiencia instrumental en algunas partes de la zona de lago y transición, para la definición de los mapas de contornos, en toda la frontera que delimita al terreno firme de la transición, se definieron las respuestas espectrales promedio de todas las estaciones de terreno firme empleadas en el estudio. Esto incluye toda la zona de lomas, además de los cerros como el de la Estrella, Santa Catarina, Tepeyac, etc. (figura 9). De igual manera, en la frontera de la zona de transición con la zona de lago, se proporcionaron dos espectros promedio, uno para un periodo de sitio de 0.8 segundos, que correspondió al promedio calculado para estaciones ubicadas en zona de transición con periodos de sitio iguales o muy cercanos a este periodo de referencia, y otro espectro promedio para un periodo del sitio de un segundo, calculado de manera similar al anterior. Esto se definió con ayuda de los mapas de isoperiodos presentados por Pérez-Rocha (1998). Las fronteras empleadas se ilustran en la figura 9. En estudios previos se han empleado procedimientos similares (Pérez-Rocha 1998, Reinoso y Ordaz 1999, Huerta y Reinoso 2002).

Algunos de los resultados más importantes del estudio se muestran en las figuras 10 a 13 , donde se presentan mapas de contornos de isodemandas de ductilidad $(\mu)$ para estructuras del grupo $\mathrm{B}$ con base en marcos de concreto reforzado considerando diferentes periodos estructurales de interés: $\mathrm{T}_{\mathrm{E}}=0.5 \mathrm{~s}$ (figura 10), $\mathrm{T}_{\mathrm{E}}=1.0 \mathrm{~s}$ (figura 11), $\mathrm{T}_{\mathrm{E}}=1.5 \mathrm{~s}$ (figura 12) y $\mathrm{T}_{\mathrm{E}}=2.0 \mathrm{~s}$, (figura 13). Los mapas mostrados están calculados considerando efectos de sobrerresistencia y tomando en cuenta los reglamentos siguientes: RCDF-42, RCDF-57 (B2), RCDF-66 (B2) y RCDF-76 (Q=4). 


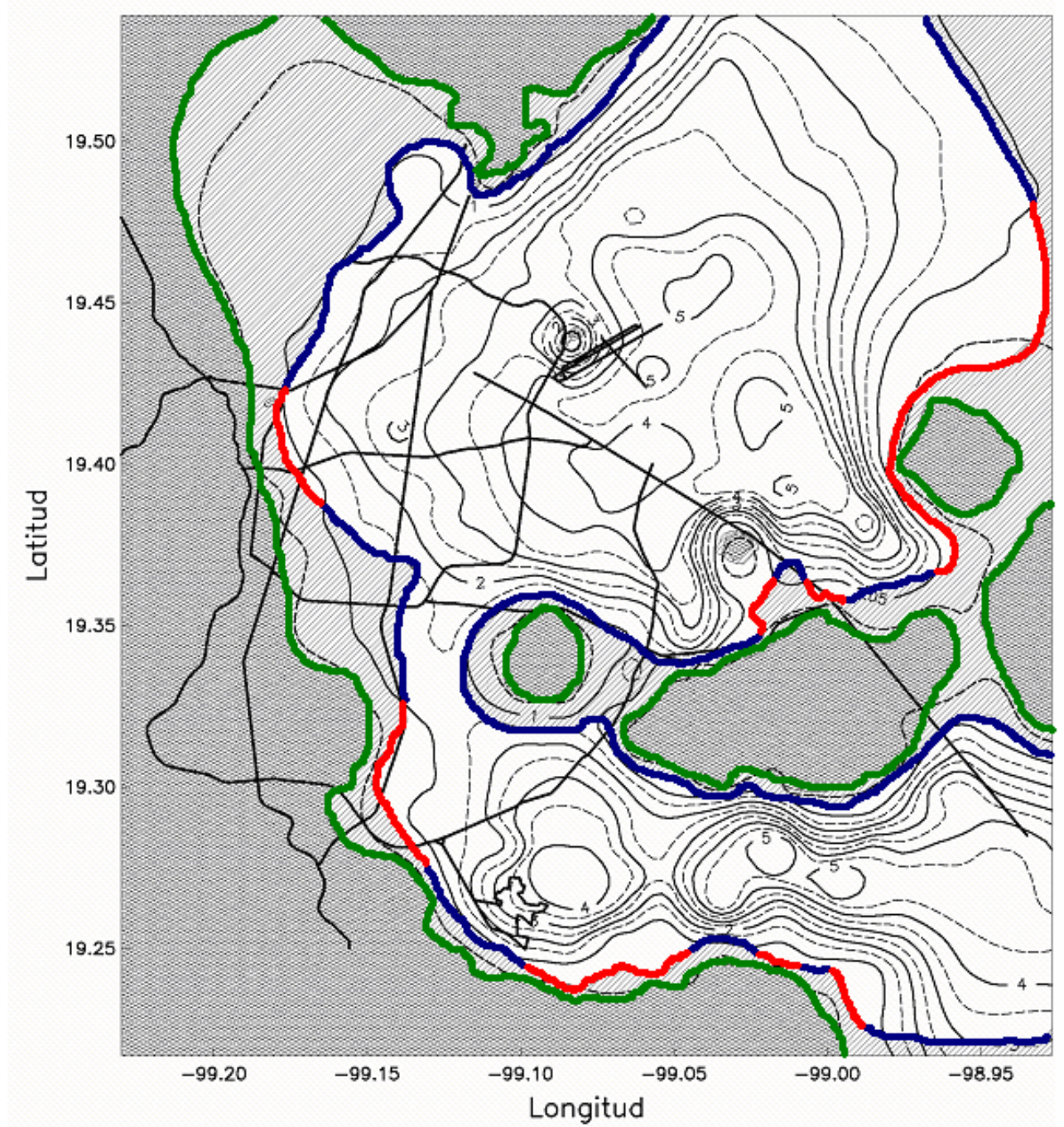

Frontera que delimita al terreno firme de la transición

Frontera que delimita a la zona de transición de la zona de lago, $\mathrm{T}_{\mathrm{s}}=1.0 \mathrm{~s}$

Frontera que delimita a la zona de transición de la zona de lago, $\mathrm{T}_{\mathrm{s}}=0.8 \mathrm{~s}$

Figura 9. Definición de fronteras de terreno firme y transición, para asignación de los valores de los espectros promedio para mejorar la estabilidad en el proceso de interpolación.

En cada uno de los mapas de isodemandas de ductilidad y desplazamiento, se muestran las zonas con mayores daños registradas durante el sismo del 19 de septiembre de 1985. Las zonas rectangulares, marcadas con línea azul, representan los límites de las zonas que presentaron daños y las otras zonas, marcadas con línea verde, dentro de las áreas rectangulares representan las zonas de mayor daño observado.

Por medio de los mapas de isodemandas que aquí se presentan, se realizó una comparación entre las zonas que presentaron daños durante el sismo del 19 de septiembre de 1985 y las zonas de mayores daños (demandas de ductilidad y desplazamiento altas) obtenidas en este estudio, esto con la finalidad de evaluar la vulnerabilidad sísmica de las estructuras de la 
zona metropolitana de la Ciudad de México. De igual manera, se valoró la confiabilidad de los resultados acorde a la densidad de instrumentación en las diferentes zonas de estudio y se identifican aquéllas zonas que presentan daño significativo y, por tanto, riesgo ante el escenario sísmico considerado en este proyecto. Lo anterior es de importancia, ya que dichas zonas pudiesen verse afectadas en un futuro por sismos de gran magnitud, como por ejemplo, el sismo que se prevé como el siguiente de gran magnitud proveniente de las costas de Guerrero.

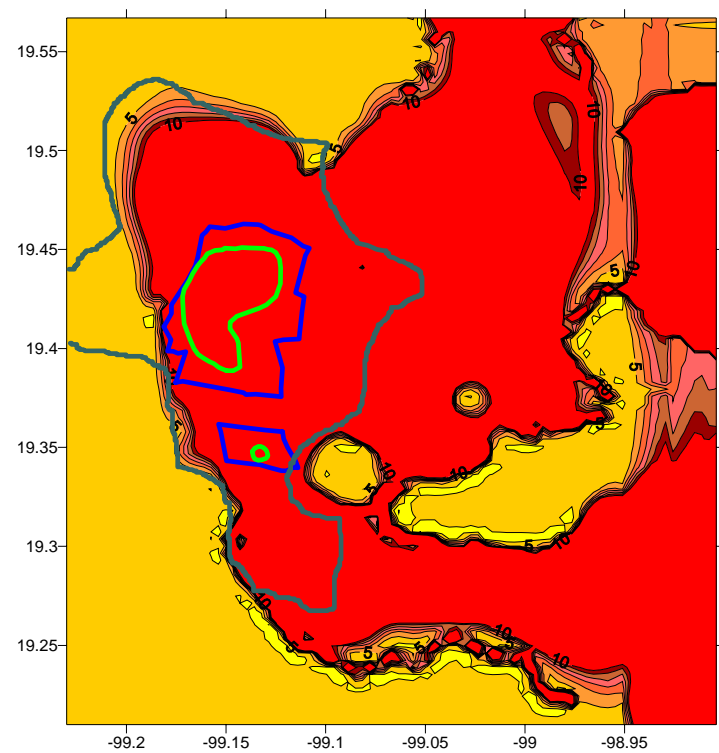

a) RCDF-1942

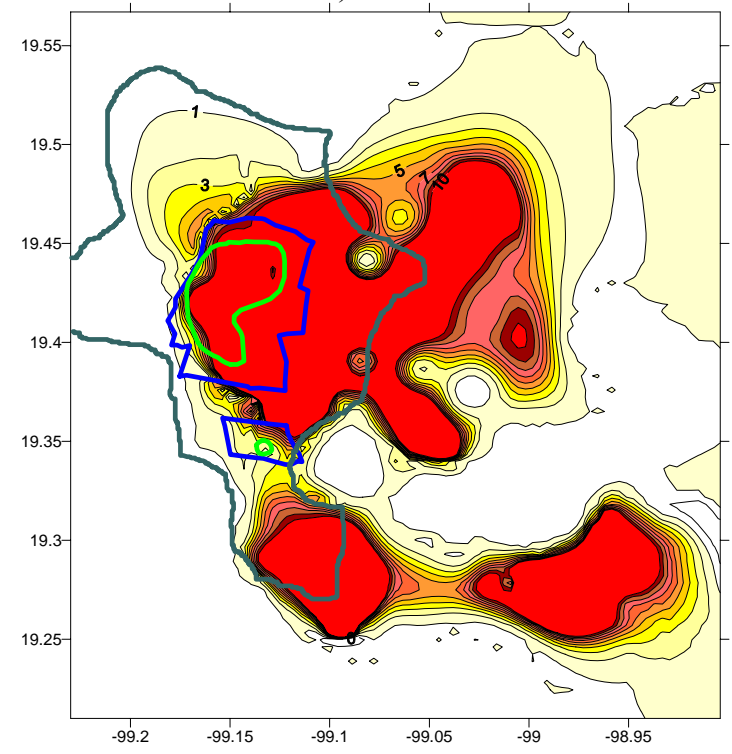

c) RCDF-1966 (B2)

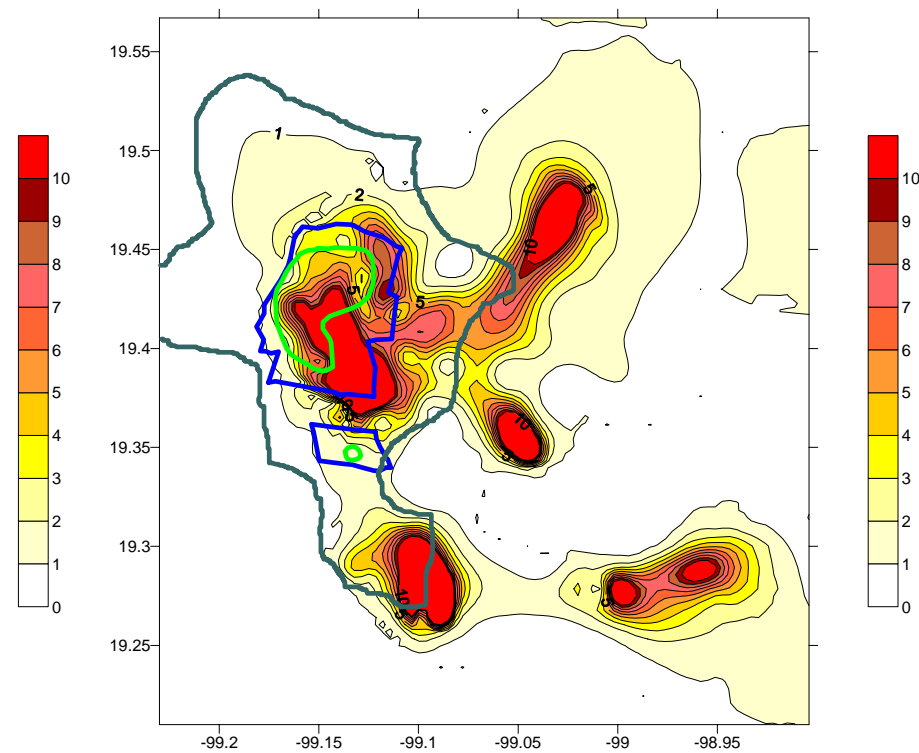

b) RCDF-1957 (B2)

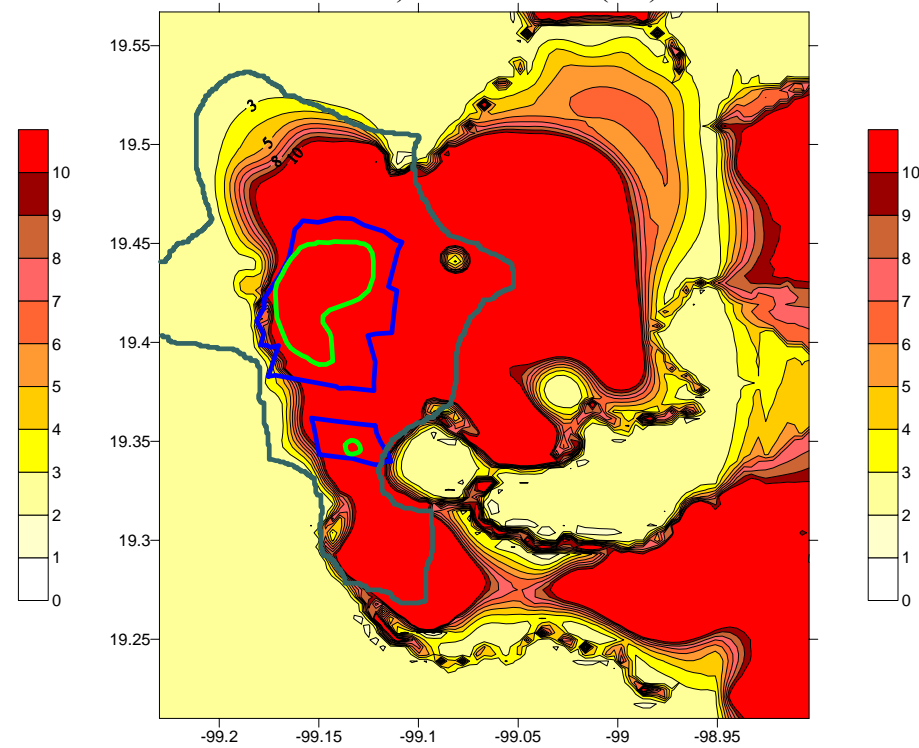

d) $\mathrm{RCDF}-1976(\mathrm{Q}=4)$

Figura 10. Mapas de isodemandas de ductilidad $(\mu)$ correspondientes a periodo estructural de $\mathrm{T}_{\mathrm{E}}=0.5 \mathrm{~s}$, considerando efectos de sobrerresistencia.

Las diferentes curvas de isodemandas de ductilidad y desplazamiento se diferencian por medio de una escala de colores. La escala que indica los niveles de demanda se muestra al 
costado derecho de cada uno de los mapas de contornos, en este caso en color blanco se presenta la respuesta dentro del intervalo elástico y la escala de colores se intensifica conforme mayores son las demandas estimadas.

Dadas las características de la red acelerométrica de la Ciudad de México (RACM, figura 2), es claro que de los mapas de contornos obtenidos, existen zonas con diferentes niveles de confiabilidad en la interpolación de los parámetros presentados (figura 14), dependiendo esto directamente de la densidad de instrumentación existente en la zona de estudio. Para las zonas cercanas a Xochimilco y Tláhuac (zona B, color verde) la densidad de instrumentación es muy baja, por lo que los contornos correspondientes a estas zonas son menos confiables que los obtenidos en la zona de mayor instrumentación (zona A, color amarillo). De igual manera, se observa que la zona $\mathrm{B}$ no es la única que tiene carencias de instrumentación, estos problemas están presentes en toda la zona $\mathrm{C}$ marcada con color rojo, la cual abarca áreas como la parte noreste de la cuidad que comprende una parte de la zona de lago y otra de transición. La zona identificada como con mejor instrumentación (zona A, figura 14) se encuentra marcada en cada uno de los mapas de contornos por medio de una línea verde gruesa. Como se observa, la zona con mayores daños y colapsos experimentados durante el sismo de septiembre de 1985 se encuentra contenida en el contorno de la zona A; por lo tanto, los contornos obtenidos en esta zona son lo suficientemente confiables para realizar una comparación con las zonas de daño observadas durante los sismos de septiembre de 1985.

Se observa de la figura 10 que para estructuras de hasta cinco niveles $\left(T_{E}=0.5 \mathrm{~s}\right)$, las demandas de ductilidad estimadas alcanzan niveles de $\mu=10$ o mayores si estas fueron diseñadas conforme a los requisitos mínimos especificados en el RCDF-42, principalmente para la zona donde se presentaron mayores daños. Esto presenta buena correlación con los datos presentados en la tabla 2 para estructuras construidas antes de 1957, donde se reportaron varios colapsos y daños graves para estructuras con base en marcos de concreto. Para estructuras diseñadas con base en los reglamentos de 1966(B2) y $1976(\mathrm{Q}=4)$ podrían esperarse colapsos o daños graves para estructuras de cinco niveles; sin embargo, durante el sismo de 1985 no existieron muchos colapsos al considerar dichos reglamentos. Cabe señalar que las menores demandas de ductilidad al considerar estructuras de cinco niveles se obtienen para el caso de estructuras diseñadas con base en el reglamento de 1957(B2), esto relacionado directamente con los requisitos mayores de resistencia estipulados en este reglamento, tal como se observa en la tabla 7.

De igual manera, en la figura 10 se observa la evolución que tuvieron los reglamentos de construcción del Distrito Federal para intentar proteger a las estructuras de este tipo con periodo fundamental $\mathrm{T}=0.5 \mathrm{~s}$ ante un evento sísmico intenso. Resulta claro que el RCDF-57 logró reducir significativamente el riesgo sísmico de este tipo de estructuras con respecto al reglamento precedente (RCDF-42), pero para el RCDF-66 se incrementa la vulnerabilidad de este tipo de estructuras, en parte debido a la disminución de los coeficientes sísmicos respecto a los estipulados en el RCDF-57, principalmente para las zonas I y III (figura 2). El RCDF-76 fue el primer reglamento mexicano en considerar criterios de resistencia última basado en espectros de diseño reducidos por concepto de deformación inelástica, por lo que al considerar estructuras diseñadas con base en este reglamento, podrían permitirse mayores demandas de ductilidad que para el RCDF-66, como se observa en la figura 10. Sin embargo, las demandas inelásticas son 
mucho mayores que aquéllas estimadas por dicho reglamento, entre otras razones porque el espectro elástico de diseño del RCDF-76 fue rebasado por los espectros de respuesta elásticos de los registros acelerométricos obtenidos en la zona de lago, particularmente los del sitio SCT, como está documentado ampliamente en la literatura. Las altas demandas de ductilidad observadas en los contornos del RCDF-76 de la figura 10 para $\mathrm{T}=0.5$ quizá detecten el colapso de algunos sistemas de marcos con base en losas planas aligeradas (tabla 2).

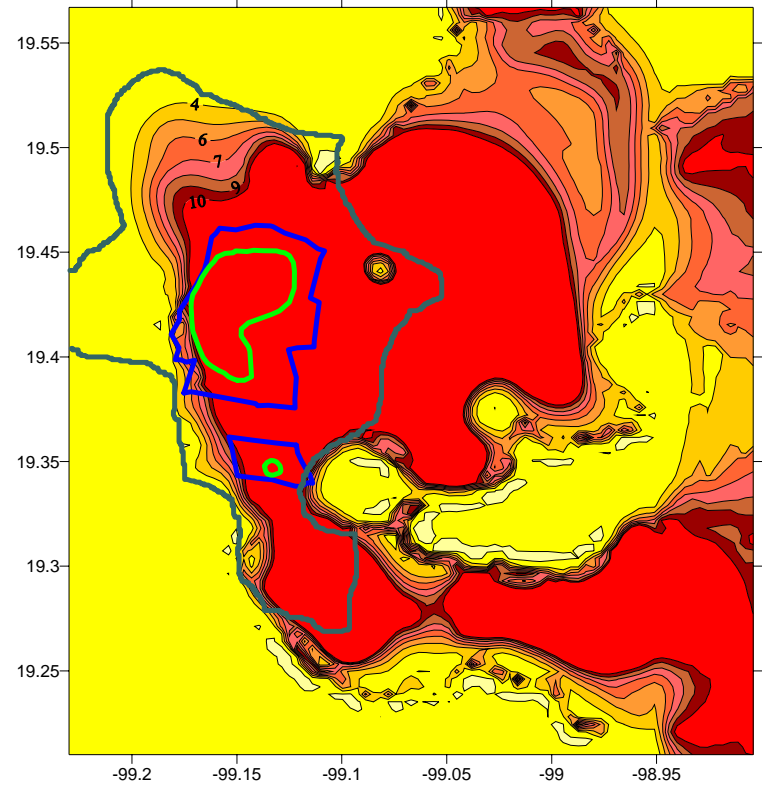

a) RCDF-1942

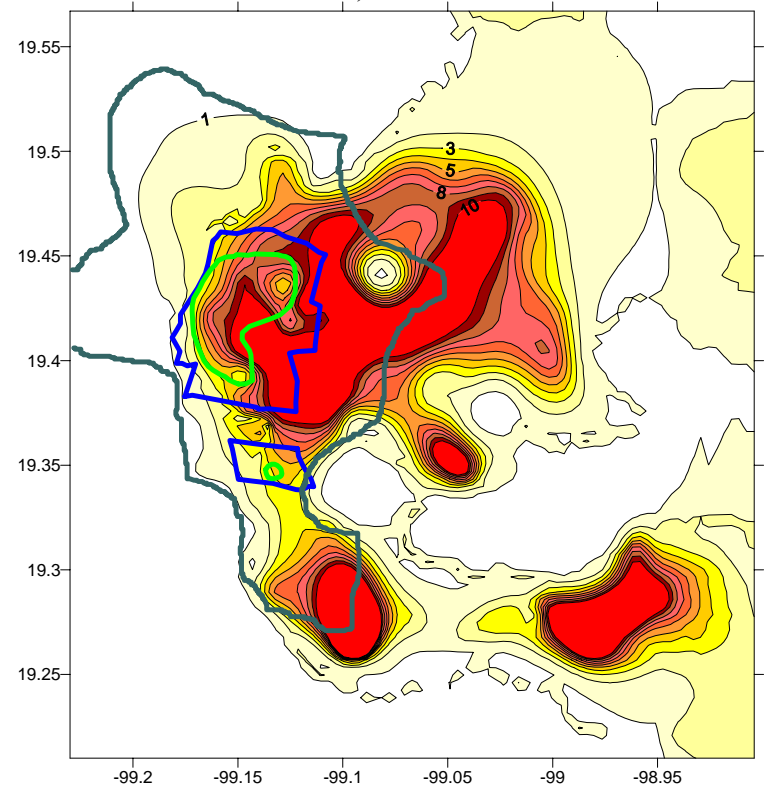

c) RCDF-1966

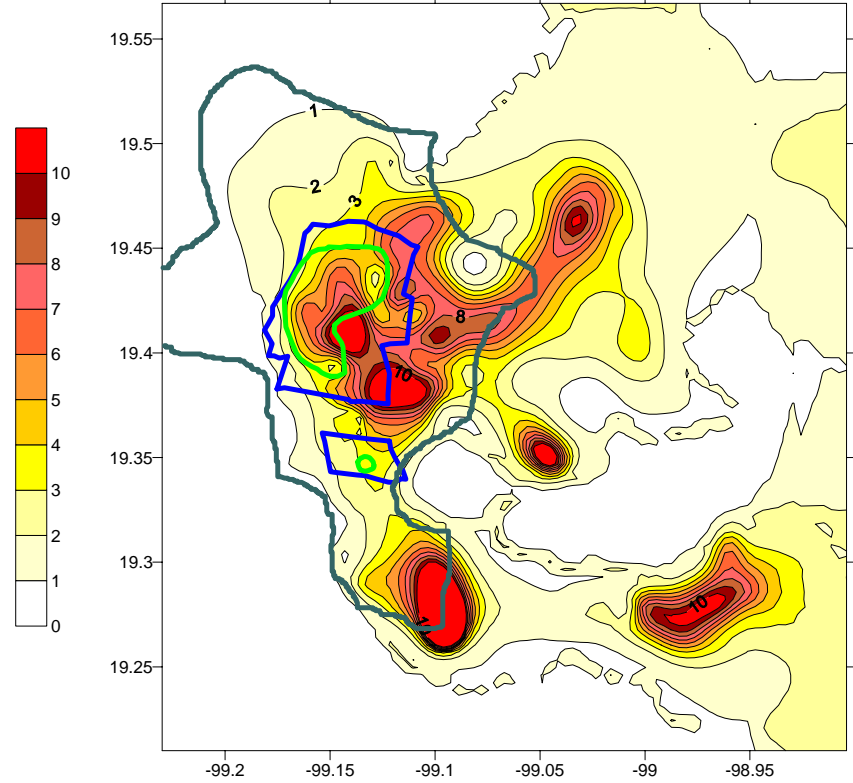

b) RCDF-1957

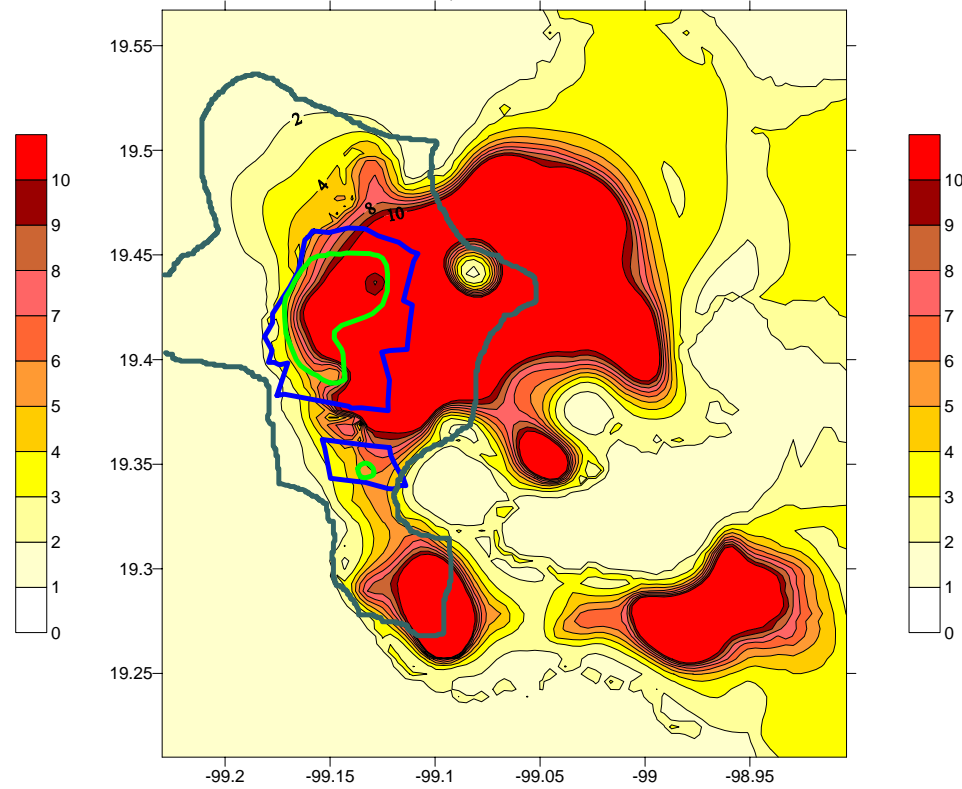

d) RCDF-1976

Figura 11. Mapas de isodemandas de ductilidad $(\mu)$ correspondientes a periodo estructural de $\mathrm{T}_{\mathrm{E}}=1.0 \mathrm{~s}$, considerando efectos de sobrerresistencia. 
Se observa a partir de la figura 11 que para estructuras con base en marcos de concreto de entre 8 y 10 niveles $\left(\mathrm{T}_{\mathrm{E}}=1 \mathrm{~s}\right)$, podrían esperarse demandas de ductilidad iguales o mayores a diez $(\mu \geq 10)$ si se consideraba un diseño conforme a lo mínimo estipulado en el RCDF-42. Afortunadamente, en septiembre de 1985 no existía un número importante de estructuras con estas características, por lo que no se presentaron niveles de daño como los mostrados en el mapa de la figura 11a.

La mayoría de las estructuras de mediana altura con base en marcos de concreto (sistemas comunes o sistemas con losa plana) fueron construidos acorde a los reglamentos de 1957, 1966 y 1976 (RCDF-57, RCDF-66, RCDF-76), por lo que esos mapas son los más importantes para observar con el fin de realizar la comparación de éstos con el reporte de daños de la tabla 2. Como ya se mencionó, las zonas de daño se encuentran identificadas con línea azul y verde en cada uno de los mapas de contornos. En la figura 11 se observan demandas de ductilidad que fluctúan entre 4 y $10(4 \leq \mu \leq 10)$ dentro de los contornos que marcan las zonas de daño de los sismos de septiembre de 1985 para cada uno de los reglamentos en cuestión (RCDF-57, RCDF66, RCDF-76), lo que nos sugiere que para los sistemas estructurales en estudio, diseñados con base en los reglamentos mencionados, podría esperarse daños graves o incluso colapsos. Lo anterior nos indica que existe una correlación razonable entre los mapas de contornos calculados y los datos reportados en la tabla 2 al considerar sistemas con base en marcos de concreto y losa plana.

Desde el punto de vista de la evolución de los reglamentos para proteger a las estructuras con base en marcos de concreto con periodos $\mathrm{T}=1 \mathrm{~s}$ ante la acción de un evento sísmico intenso, se puede llegar a conclusiones semejantes a las elaboradas para estructuras con periodo $\mathrm{T}=0.5 \mathrm{~s}$, es decir, el RCDF-57 reduce significativamente la vulnerabilidad con respecto al RCDF-42; sin embargo, los reglamentos de 1966 y 1976 incrementaron la vulnerabilidad para este tipo de estructuras. Aunque existen similitudes en cuanto a los niveles de demandas de ductilidad para estos dos reglamentos, el RCDF-76 presentaba mejores requerimientos en cuanto al detallado que reglamentos anteriores (RCDF-57 y RCFD-66). Esto puede explicar el por qué del menor número de estructuras con daños graves y colapsos cuyos diseños estaban acorde al RCDF-76, respecto a las diseñadas con base en reglamentos previos (RCDF-57 y RCDF-66, tabla 2).

Se observa de la figura 12 que para estructuras con base en marcos de concreto de entre 13 y 15 niveles $\left(\mathrm{T}_{\mathrm{E}}=1.5 \mathrm{~s}\right)$, podrían esperarse demandas de ductilidad iguales o mayores a diez $(\mu \geq 10)$ si se consideraba un diseño conforme a lo mínimo estipulado en el RCDF-42. Afortunadamente, en septiembre de 1985 no existía un número importante de estructuras con estas características, por lo que no se presentaron niveles de daño como los mostrados en el primer mapa de la figura 12.

La mayoría de las estructuras de mediana altura con base en marcos de concreto fueron construidos acorde a los reglamentos de 1957, 1966 y 1976 (RCDF-57, RCDF-66, RCDF-76), por lo que debe realizarse una comparación de los mapas calculados con base en cada uno de estos reglamentos con los reporte de daños de la tabla 2. De la observación de la figura 12 resulta claro que los niveles de demandas de ductilidad para este periodo son considerablemente menores a las estimadas para periodos menores (figuras 10 y 11), lo que sugiere que los niveles de daño 
esperado para el mismo inventario de estructuras deben ser menores dentro de la zona de interés. En efecto, se observa de la tabla 2 un menor número de estructuras de concreto de 13 a 15 pisos (marcos de concreto y losa plana) que contaban con niveles de daño severo o colapso. Ciertamente, existía en septiembre de 1985 un menor inventario de estructuras con este número de pisos que estructuras entre 8 y 10 niveles, como se infiere a partir de la información proporcionada en la tabla 1. Por lo tanto, la correlación entre los mapas de contornos y el mapa de daños de los sismos de septiembre de 1985 se considera razonable.

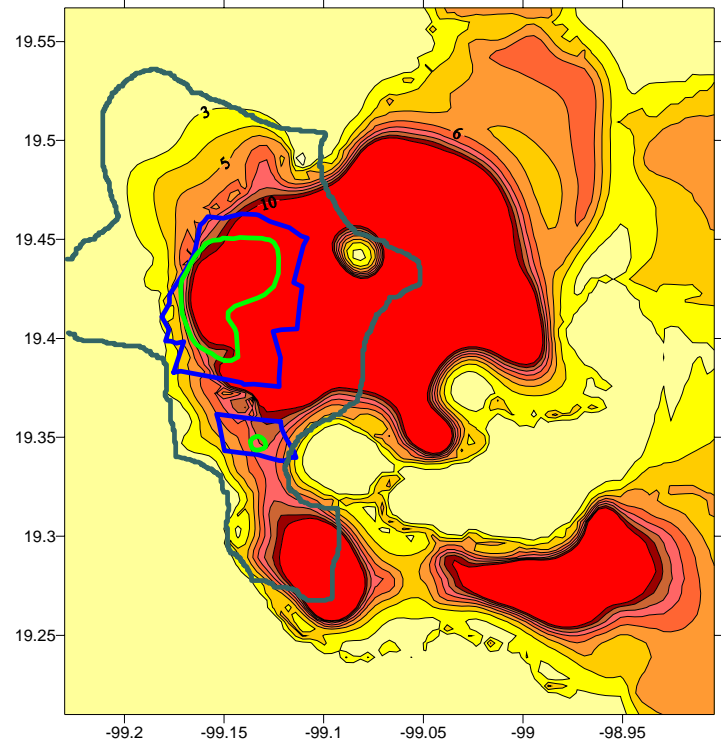

a) RCDF-1942

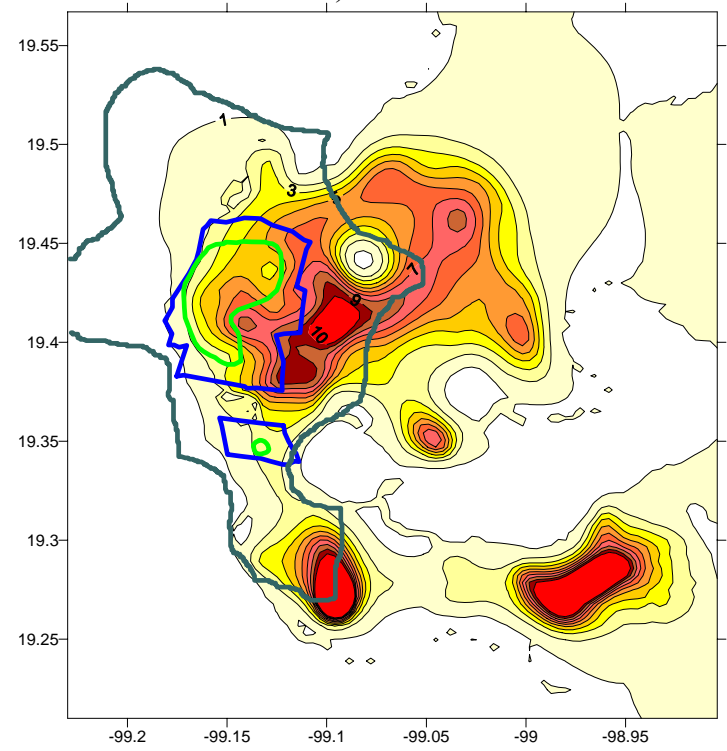

c) $\mathrm{RCDF}-1966$

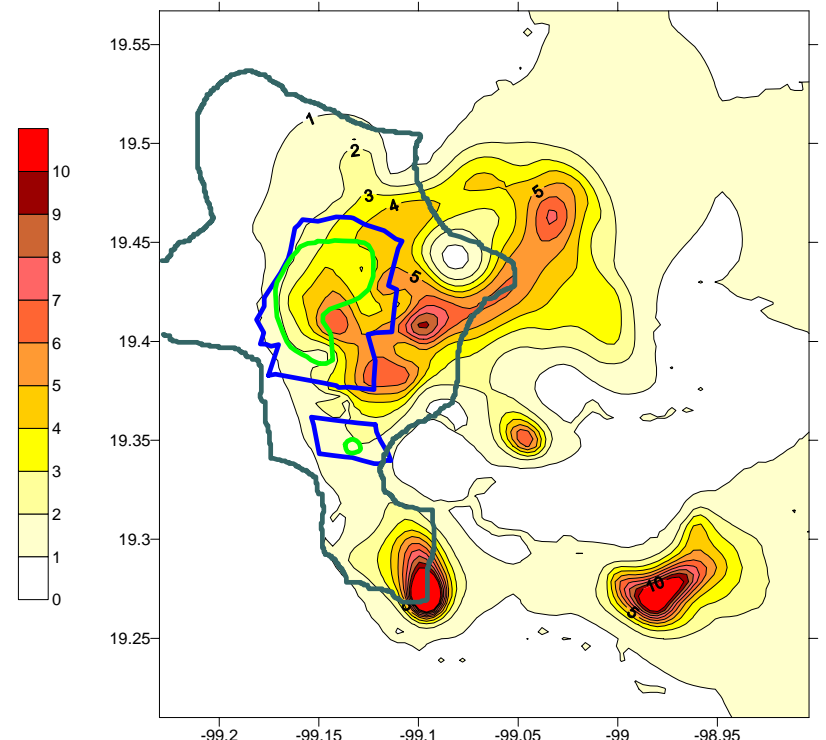

b) RCDF-1957

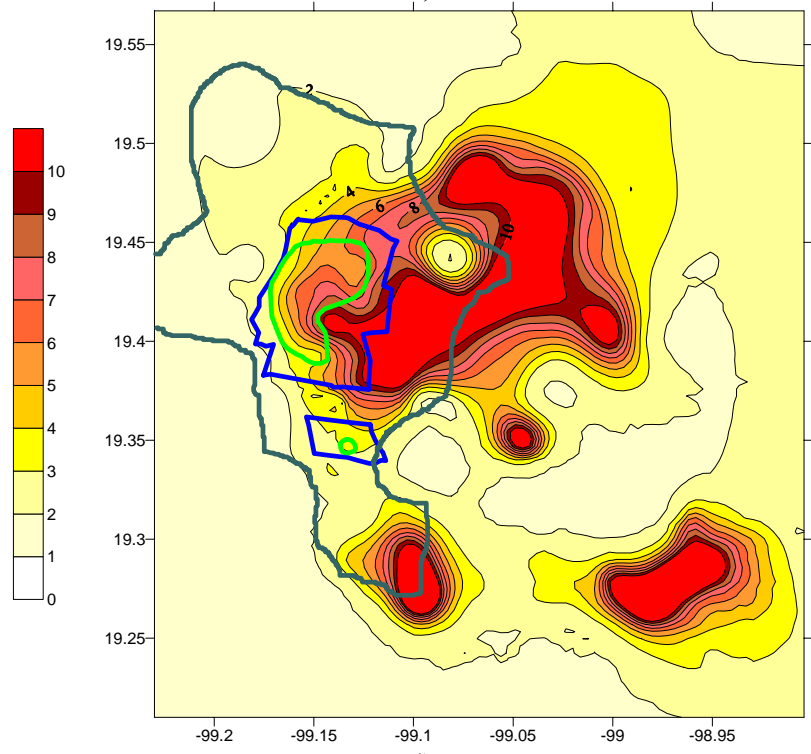

d) RCDF-1976

Figura 12. Comparación entre mapas de isodemandas de ductilidad correspondientes a periodo estructural de $\mathrm{T}_{\mathrm{E}}=1.5 \mathrm{~s}$, considerando efectos de sobrerresistencia. 
De la figura 13, correspondiente a periodo de $\mathrm{T}_{\mathrm{E}}=2.0$ segundos (estructuras con 15 niveles o más), se observa la variación que las demandas de ductilidad presentan acorde al reglamento de construcción considerado.

Es claro a partir de la observación de la figura 13 que, al considerar el reglamento de 1942, las demandas son extremadamente altas en varias zonas, como ya se ha observado para el caso de otros periodos estructurales. Lo anterior se debe a las bajas resistencias estipuladas en dicho reglamento, $\mathrm{V}=0.025 \mathrm{~W}$. Sin embargo, en septiembre de 1985 no existían estructuras con estas características diseñadas conforme a ese reglamento, por lo que afortunadamente no se presentaron los niveles de daño mostrados en el mapa de contornos correspondiente al RCDF-42. De igual manera, se observa que los niveles de demandas de ductilidad se redujeron significativamente al considerar un diseño con base en el reglamento de 1957 respecto a uno con base en el RCDF-42; por ende, existió una reducción de la vulnerabilidad de este tipo de estructuras. El mapa correspondiente al RCDF-66 presenta grandes similitudes respecto al calculado con base en el RCDF-57. Al comparar el mapa obtenido con base en el RCDF-76 respecto al calculado con base en el RCDF-66, se observan mayores niveles de demandas para el primer caso, aunque debe considerarse que en este reglamento se establecen mejores requisitos de detallado para marcos de concreto, lo que probablemente permitió a dichas estructuras a desarrollar mayores niveles de ductilidad que a las diseñadas con base en el reglamento de 1966.

Al considerar el reglamento de 1976, se observan demandas de ductilidad mucho menores tanto en la zona de terreno firme como en la zona de transición, respecto a las estimadas para periodos estructurales menores $\left(\mathrm{T}_{\mathrm{E}}=0.5 \mathrm{~s}, \mathrm{~T}_{\mathrm{E}}=1.0 \mathrm{~s}, \mathrm{~T}_{\mathrm{E}}=1.5 \mathrm{~s}\right)$. Para la zona de lago existen pequeñas zonas donde aún los niveles de demandas son muy elevados. Dichas zonas no se encuentran dentro del mapa que delimita los daños ocurridos durante los sismos de septiembre de 1985, lo que nos indica que existen zonas vulnerables diferentes a las dañadas previamente por sismos de gran magnitud, como los ocurridos en 1957, 1976 y 1985. Dichas zonas pueden considerarse como de alto peligro sísmico si se considera que las probabilidades de la ocurrencia de un evento sísmico de gran magnitud en un futuro no son despreciables, principalmente considerando que la brecha de Guerrero, según los expertos, será la zona donde se genere el próximo sismo de gran magnitud, cuyo tamaño puede incluso superar al del 19 de septiembre de 1985.

Este gran sismo puede ocasionar daños severos en la zona metropolitana de la Ciudad de México, principalmente si no se toman en cuanta factores como los que se muestran en los mapas de demandas, es decir, que pueden existir zonas diferentes a las ya conocidas con un importante peligro sísmico, tales como lo es la zona oriente cercana al aeropuerto, ciudad Nezahualcoyotl (oriente) y la zona sur-suroriente correspondiente a los antiguos lagos de Xochimilco y ChalcoTláhuac, las cuales, como se observa de la figura 13, pueden presentar demandas de ductilidad de importancia. Sin embargo, como ya se mencionó, la deficiente instrumentación en estas zonas no permite tener la definición necesaria para llegar a conclusiones más contundentes con respecto al peligro sísmico y la vulnerabilidad de estructuras en estas zonas. Como se mencionó, en las figuras 10 a 13 se identifica también a la zona que se considera con mayor nivel de instrumentación, marcada con línea verde (zona en color amarillo de la figura 14). 


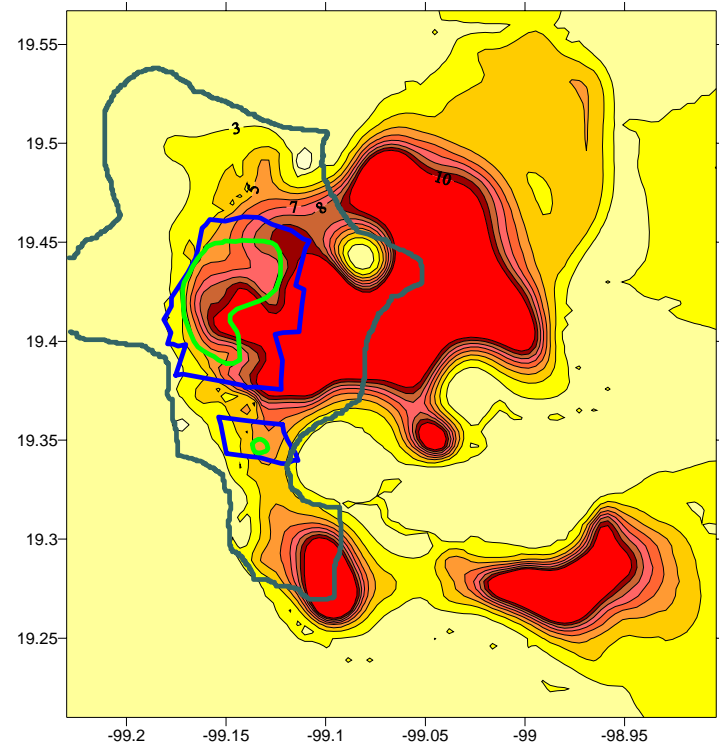

a) RCDF-1942

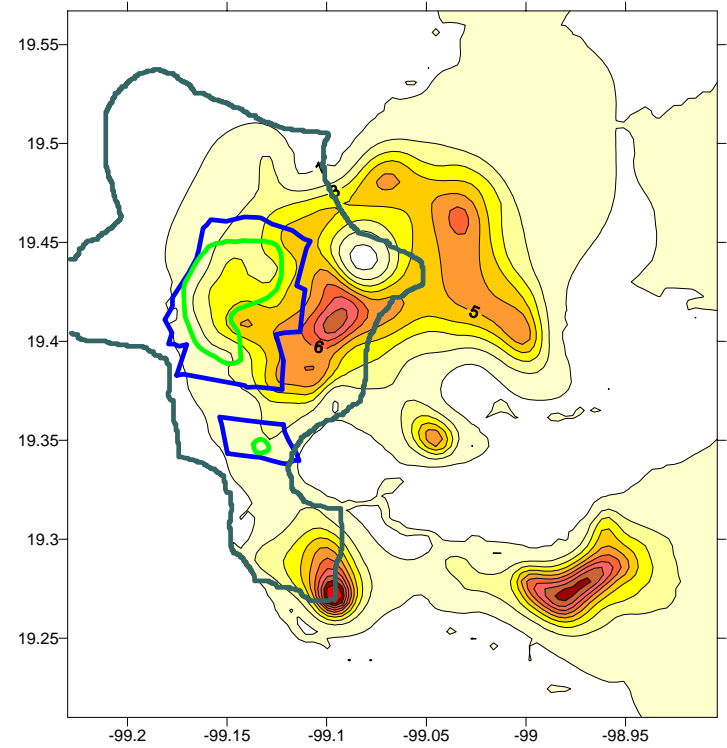

c) RCDF-1966

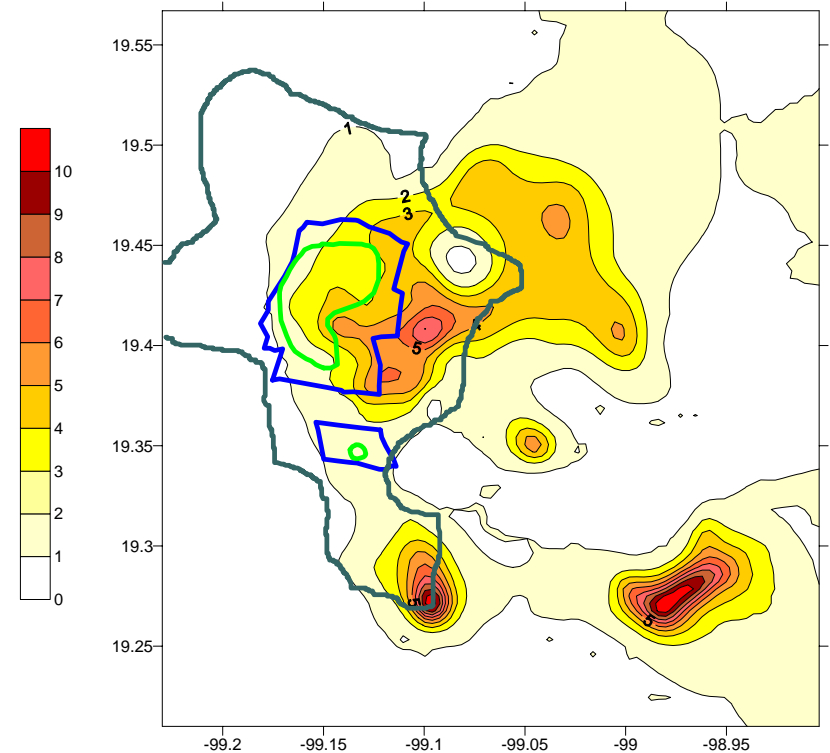

b) RCDF-1957

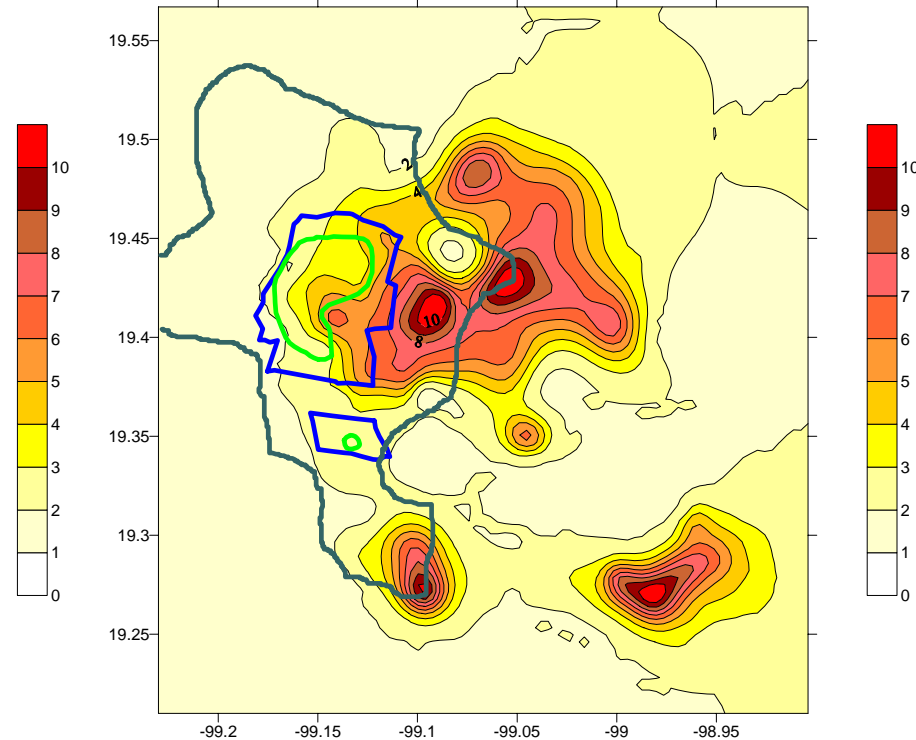

d) RCDF-1976

Figura 13. Comparación entre mapas de isodemandas de ductilidad correspondientes a periodo estructural de $\mathrm{T}_{\mathrm{E}}=2.0 \mathrm{~s}$, considerando efectos de sobrerresistencia.

Finalmente, en la figura 15 se presentan mapas de isodemandas de desplazamiento inelástico para estructuras diseñadas con base en el Reglamento de $1976(\mathrm{Q}=4)$ y considerando un factor de sobrerresistencia de dos. De la figura se observan las variaciones en dicho parámetro al considerar diferentes periodos estructurales $\left(\mathrm{T}_{\mathrm{E}}=0.5 \mathrm{~s}, \mathrm{~T}_{\mathrm{E}}=1.0 \mathrm{~s}, \mathrm{~T}_{\mathrm{E}}=1.5 \mathrm{~s}, \mathrm{~T}_{\mathrm{E}}=2.0 \mathrm{~s}\right)$. Se observa que para periodo estructural de un segundo, los niveles de demandas en las zonas con mayores desplazamientos fluctúan entre los 60 y los $75 \mathrm{~cm}$ para la mayor parte de la zona de lago; entre los ocho y los $15 \mathrm{~cm}$ para la zona de transición, y de uno a seis en la zona de terreno firme. Las mayores demandas para la zona de lago corresponden a las áreas cercanas a Xochimilco y Tláhuac, las cuales presentan demandas de desplazamiento mayores a $100 \mathrm{~cm}$. 


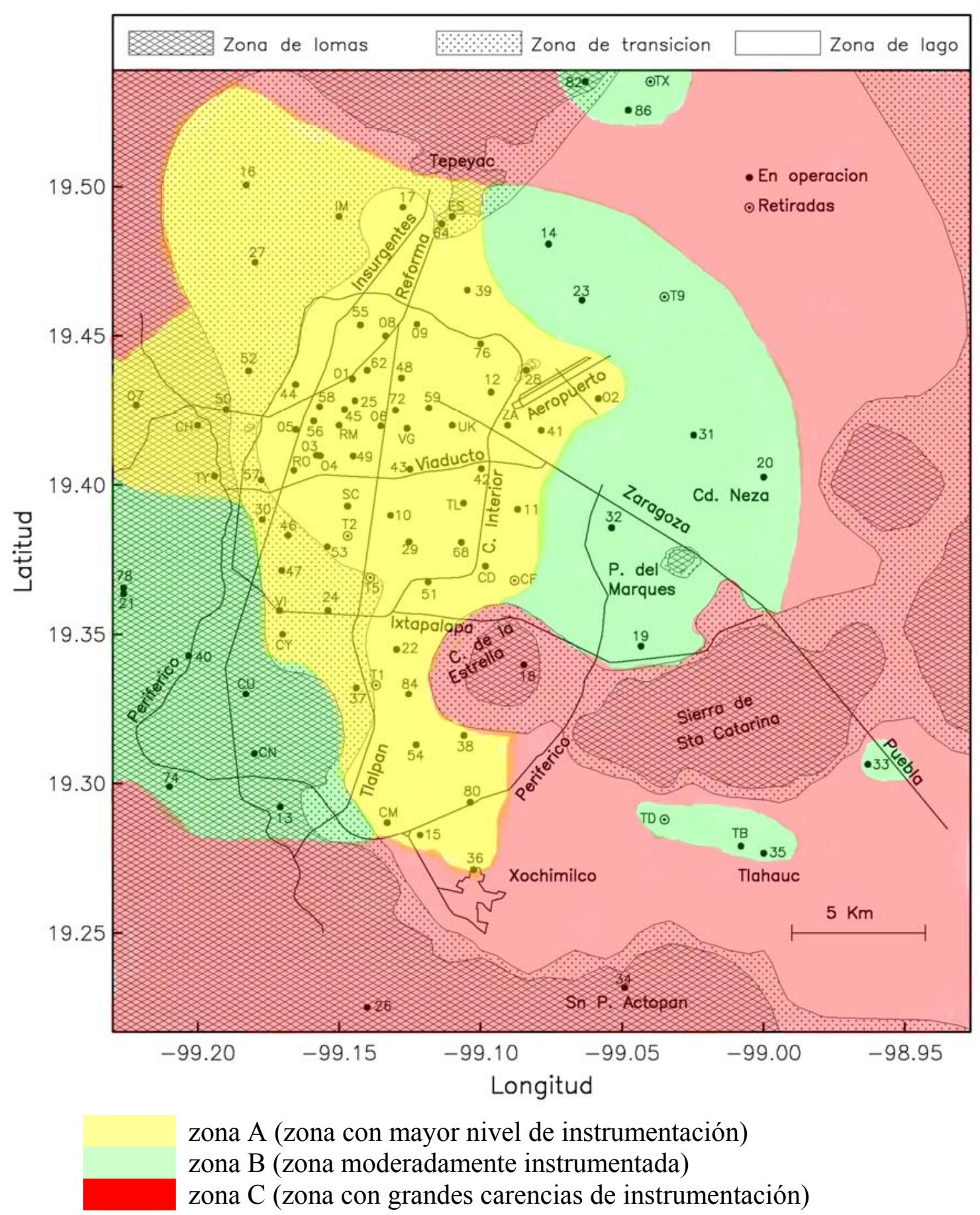

Figura 14. Identificación de las zonas con mayor y menor densidad de instrumentación sísmica

\section{CONCLUSIONES}

Los mapas calculados con base en los reglamentos de construcciones de 1957 y 1966 muestran claramente el efecto que tuvieron las disposiciones reglamentarias en estructura con base en 
marcos de concreto reforzado, particularmente en los cambios de los coeficientes sísmicos de diseño. En efecto, las resistencias estipuladas en el Reglamento de 1966 fueron, en general, reducidas para los grupos B1 y B2 con respecto a las estipuladas en 1957, sobre todo para las zonas I y III (tabla 6). Este cambio se puede señalar en los mapas de isodemandas de ductilidad calculados, al comparar aquéllos calculados con base en los reglamentos de 1957 y 1966 . Los mapas calculados con base en el Reglamento de 1966 muestran áreas mayores con niveles de demandas de ductilidad elevados que aquéllos calculados con base en el Reglamento de 1957.

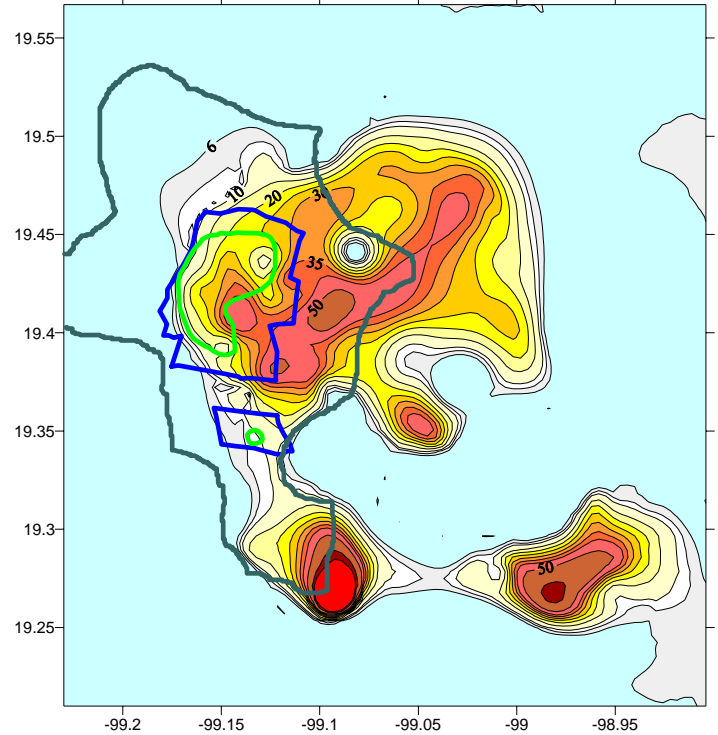

a) $\mathrm{T}_{\mathrm{E}}=0.5 \mathrm{~s}$

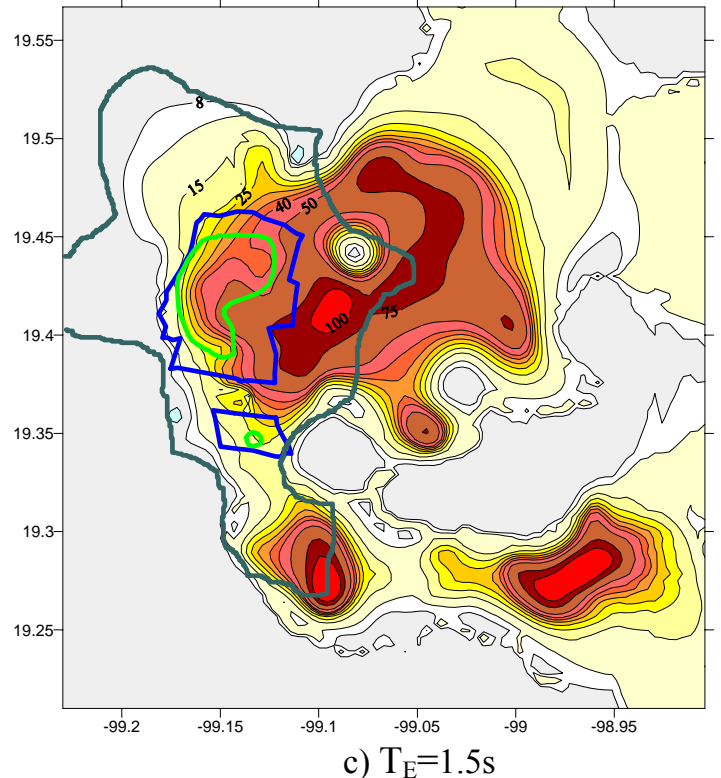

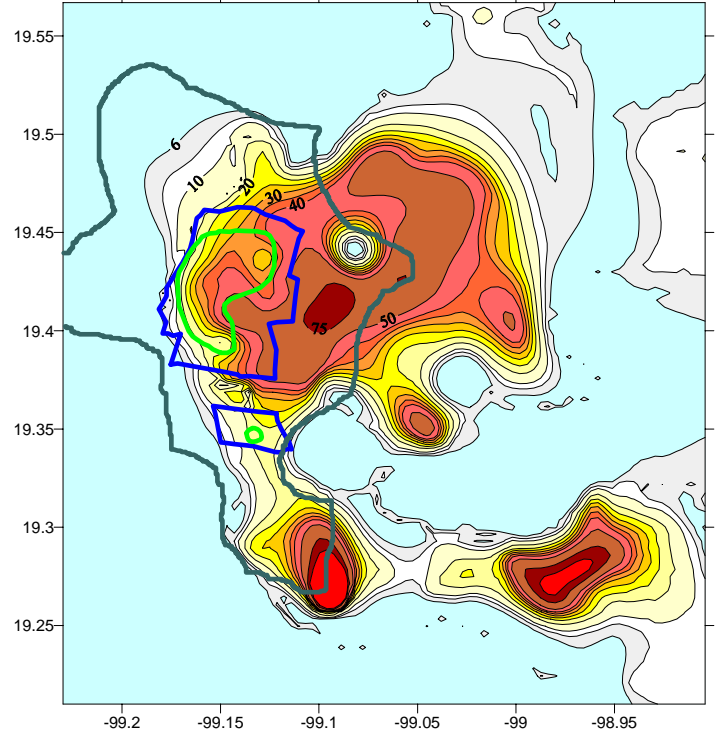

b) $\mathrm{T}_{\mathrm{E}}=1.0 \mathrm{~s}$

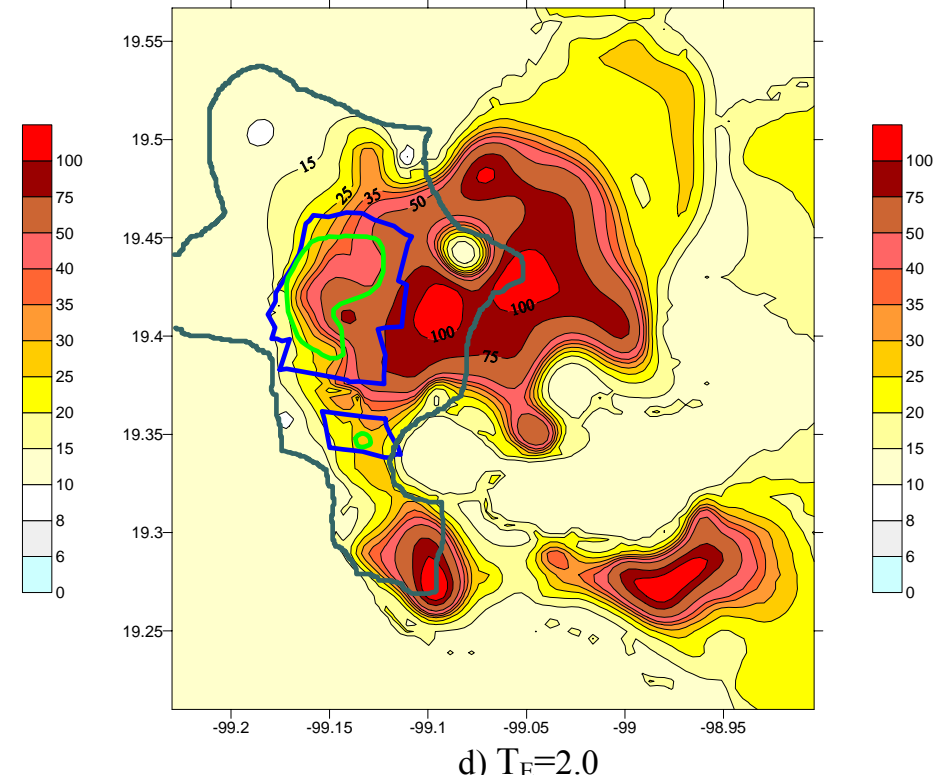

Figura 15. Mapas de isodemandas de desplazamiento inelástico (cm) para estructuras diseñadas con base en el RCDF-76, $\mathrm{Q}=4$, considerando efectos de sobrerresistencia. 
Para todos los reglamentos en estudio, se observó que al considerar los efectos de la sobrerresistencia, existen disminuciones importantes en los niveles de demandas de ductilidad con respecto a las calculadas con base en resistencias nominales (no mostrado, Godínez 2005). Para el caso de las demandas de desplazamiento, este efecto en particular es notorio al considerar un periodo estructural de $\mathrm{T}_{\mathrm{E}}=0.5 \mathrm{~s}$ (estructuras de poca altura). Se observó de igual manera que en ciertas ocasiones, aún considerando efectos de sobrerresistencia, los desplazamientos se incrementan con respecto a los calculados con base en las resistencias nominales (Godínez 2005).

Para la mayoría de los casos, al considerar periodos estructurales de $\mathrm{T}_{\mathrm{E}}=0.5 \mathrm{~s}$ y $\mathrm{T}_{\mathrm{E}}=1.0 \mathrm{~s}$, se observó una muy buena correlación entre el mapa de daños correspondiente a los sismos de septiembre de 1985 y las zonas con las máximas demandas de los mapas de contornos calculados con la metodología propuesta. Cuando se consideran periodos estructurales mayores $\left(\mathrm{T}_{\mathrm{E}}=1.5 \mathrm{~s}\right.$ y $\mathrm{T}_{\mathrm{E}}=2.0 \mathrm{~s}$ ), la correlación que se obtiene es razonable, ya que aunque en varios casos el mapa de daños de los sismos de septiembre de 1985 no coincide con las zonas de demandas máximas, si coincide con contornos en que las demandas de ductilidad varían entre cuatro y cinco, lo cual para la mayoría de los reglamentos en evaluación implica daño en las estructuras, debido a que en los reglamentos de 1942, 1957 y 1966 no se especificaban los requerimientos necesarios, especialmente en el detallado, para desarrollar niveles de ductilidad como los calculados para esta zona. Por esta razón, se considera que la metodología planteada puede ser empleada de manera confiable para identificar las zonas con mayores demandas para diferentes sistemas estructurales ante un escenario sísmico postulado.

Se observó en la mayoría de los mapas de isodemandas, que para ambos componentes, los contornos de mayores demandas de ductilidad y desplazamiento se ubican en la zona al suroriente del aeropuerto, en Ciudad Nezahualcoyotl y en los antiguos lagos de Xochimilco y Chalco-Tláhuac. Esto indica la imperiosa necesidad, en caso de confirmarse lo anterior con estudios apoyados en mediciones experimentales en las zonas identificadas con grandes carencias de instrumentación (figura 14), de clasificarlas en el futuro cercano como zonas de alto peligro sísmico en el valle de México. Estas zonas, particularmente Xochimilco y Tláhuac, han sido previamente identificadas como susceptibles de presentar a) altas amplificaciones espectrales (Reinoso y Ordaz 1999) y, b) grandes niveles de energía de entrada (Huerta y Reinoso 2002). Por lo tanto, debe hacerse un comentario de alerta para estas zonas, como lo hicieran Reinoso y Ordaz (1999) para las nuevas urbanizaciones en Xochimilco, y tomar las debidas precauciones en futuros proyectos de urbanización que involucren a estas zonas.

Se reconoce que aunque este estudio representa un paso adelante con respecto a estudios de vulnerabilidad previos realizados para la zona metropolitana del valle de México, aún se tienen varias limitaciones para valorar globalmente la vulnerabilidad de distintos sistemas estructurales, principalmente los asociados a la deficiente instrumentación sísmica que dispone el valle de México en su zona oriente y en las inmediaciones de Xochimilco y Tláhuac. Sin embargo, el estudio es aún así valioso para potenciar los contornos de mayores demandas de sismos intensos que afecten al valle de México con la directividad más frecuentemente observada. 
Sería recomendable que para aquéllas zonas en que la densidad de instrumentación es baja, se realizaran programas de densificación instrumental, lo cual sería útil para corroborar si, en efecto, los niveles de ductilidad y desplazamiento en estas zonas son del orden que se muestra en los mapas de contornos presentados en este trabajo. Esto permitiría tener mayor certidumbre sobre las zonas donde se presentan mayores demandas y, por tanto, esta información sería más confiable en la planeación urbana y uso del suelo de la zona metropolitana de la Ciudad de México.

Con el fin de complementar el estudio, deberían confrontarse estos contornos con un mapa del censo de estructuras de estas características ubicadas espacialmente en la ciudad, para cuantificar en qué zonas existía una densidad importante de este tipo de edificios, y en qué zonas ni siquiera existían edificios de este tipo. De igual manera, debería de complementarse este estudio mediante la comparación y cruce de información proveniente de proyectos que evalúen otros parámetros de interés, como pueden ser, la energía de entrada, velocidades máximas, $\mathrm{y}$ aceleraciones máximas, entre otras.

Finalmente, mediante la metodología propuesta podría realizarse la evaluación de los reglamentos de construcción posteriores a los sismos de septiembre de 1985, es decir, los correspondientes a 1987, 1993 y 2004, considerando los escenarios sísmicos contemplados en la definición de los espectros de diseño de dichos reglamentos, que son los considerados en el estudio de Pérez-Rocha (1998), para los principales sistemas estructurales establecidos en dichos cuerpos normativos.

\section{AGRADECIMIENTOS}

El primer autor agradece la beca que Conacyt le otorgó para desarrollar sus estudios de maestría en la Universidad Autónoma Metropolitana Azcapotzalco (UAM-A), así como la beca complementaria que la UAM-A le otorgó para completar su proyecto de tesis.

\section{REFERENCIAS}

BMDSF (2000), "Base Mexicana de Datos de Sismos Fuertes volumen 2", CDROM, Sociedad Mexicana de Ingeniería Sísmica, A.C., diciembre.

Correa, H (2005), "Evaluación de los criterios de diseño sísmico de la propuesta de Reglamento de Construcciones para el Distrito Federal (RCDF-2001) para edificios regulares con base en marcos dúctiles de concreto reforzado", Tesis de Maestría, División de Estudios de Posgrado de la Facultad de Ingeniería, Universidad Nacional Autónoma de México, mayo.

"Fundación ICA" (1988), Experiencias derivadas de los sismos de septiembre de 1985, primera edición, Limusa, México.

Godínez, E A (2005), “Evaluación de la vulnerabilidad sísmica de estructuras existentes en el Distrito Federal. El caso específico del sismo del 19 de septiembre de 1985", Tesis de Maestría, Posgrado en Ingeniería Estructural, División de Ciencias Básicas e Ingeniería, Universidad Autónoma Metropolitana Azcapotzalco, mayo. 
Gómez, A, H Juárez y J Iglesias (1991), "Intensidades y demandas de ductilidad de sismos recientes en la ciudad de México”, Revista de Ingeniería Sísmica, SMIS, No. 43, pp. 3-18.

Huerta, B y E Reinoso (2002), "Espectros de energía de movimientos fuertes registrados en México", Revista de Ingeniería Sísmica, SMIS, No 66, pp. 45-72.

"Instituto de Ingeniería, UNAM" (1985), "La UNAM ante los sismos del 19 de septiembre de 1985”, Informe Preliminar, México, octubre.

Murià, D y R González (1995), "Propiedades dinámicas de edificios de la Ciudad de México", Revista de Ingeniería Sísmica, SMIS, No. 51, pp. 25-45.

NTCS-04 (2004) "Normas Técnicas Complementarias para Diseño por Sismo”, Gaceta Oficial del Distrito Federal, Tomo II, No. 103-BIS, octubre, pp. 55-77.

Pérez-Rocha, L E (1998), "Respuesta sísmica estructural: efectos de sitio e interacción sueloestructura (aplicaciones al valle de México)", Tesis Doctoral, División de Estudios de Posgrado de la Facultad de Ingeniería, Universidad Nacional Autónoma de México.

Pineda, O y M Ordaz (2004), "Mapas de velocidad máxima del suelo para la Ciudad de México", Revista de Ingeniería Sísmica, SMIS, No. 71, pp. 37-62.

Reinoso, E (1991), "Efectos sísmicos locales en el Valle de México: amplificación medida en la zona lacustre”, Memorias, IX Conferencia Nacional de Ingeniería Sísmica, Manzanillo, México, Vol. II, pp. 224-236.

Reinoso, E y M Ordaz (1999), "Spectral rations for Mexico City from free-field recordings", Earthquake Spectra, Vol. 15, No. 2, pp 273-295.

Saiidi, M y M A Sozen (1979), "Simple and complex models for nonlinear seismic response of reinforce concrete", Civil Engineering Studies, Structural Research Series No. 465, University of Illinois at Urbana-Champaign, agosto.

Tena, A (1998), "Evaluación sísmica simplificada de estructuras existentes", Revista de Ingeniería Sísmica, SMIS, No 59, pp. 1-36.

Tena-Colunga, A (2001), "Displacement ductility demand spectra for the seismic evaluation of structures”, Engineering Structures, Vol. 23, No. 10, pp. 1319-1330. 